ARTICLE

DOI: $10.1038 / \mathrm{s} 41467-018-03917-2$

\title{
Genome-wide and high-density CRISPR- Cas9 screens identify point mutations in PARP1 causing PARP inhibitor resistance
}

\author{
Stephen J. Pettitt (10) 1,2, Dragomir B. Krastev 1,2, Inger Brandsma1,2, Amy Dréan ${ }^{1,2}$, Feifei Song ${ }^{1,2}$, \\ Radoslav Aleksandrov ${ }^{3}$, Maria I. Harrell4, Malini Menon 1,2, Rachel Brough1,2, James Campbell,2, \\ Jessica Frankum ${ }^{1,2}$, Michael Ranes ${ }^{5}$, Helen N. Pemberton ${ }^{1,2}$, Rumana Rafiq ${ }^{1,2}$, Kerry Fenwick ${ }^{6}$, Amanda Swain ${ }^{6}$, \\ Sebastian Guettler (1) ${ }^{5}$, Jung-Min Lee ${ }^{7}$, Elizabeth M. Swisher ${ }^{4}$, Stoyno Stoynov ${ }^{3}$, Kosuke Yusa ${ }^{8}$, \\ Alan Ashworth ${ }^{9} \&$ Christopher J. Lord ${ }^{1,2}$
}

Although PARP inhibitors (PARPi) target homologous recombination defective tumours, drug resistance frequently emerges, often via poorly understood mechanisms. Here, using genome-wide and high-density CRISPR-Cas9 "tag-mutate-enrich" mutagenesis screens, we identify close to full-length mutant forms of PARP1 that cause in vitro and in vivo PARPi resistance. Mutations both within and outside of the PARP1 DNA-binding zinc-finger domains cause PARPi resistance and alter PARP1 trapping, as does a PARP1 mutation found in a clinical case of PARPi resistance. This reinforces the importance of trapped PARP1 as a cytotoxic DNA lesion and suggests that PARP1 intramolecular interactions might influence PARPimediated cytotoxicity. PARP1 mutations are also tolerated in cells with a pathogenic BRCA1 mutation where they result in distinct sensitivities to chemotherapeutic drugs compared to other mechanisms of PARPi resistance (BRCA1 reversion, 53BP1, REV7 (MAD2L2) mutation), suggesting that the underlying mechanism of PARPi resistance that emerges could influence the success of subsequent therapies.

\footnotetext{
${ }^{1}$ The CRUK Gene Function Laboratory, The Institute of Cancer Research, London SW3 6JB, UK. ${ }^{2}$ Breast Cancer Now Toby Robins Research Centre, The Institute of Cancer Research, London SW3 6JB, UK. ${ }^{3}$ Institute of Molecular Biology "Roumen Tsanev", Bulgarian Academy of Sciences, Sofia 1113, Bulgaria. ${ }^{4}$ University of Washington School of Medicine, 1959 NE Pacific St, Seattle, WA 98195, USA. ${ }^{5}$ Divison of Structural Biology, The Institute of Cancer Research, London SW3 6JB, UK. ${ }^{6}$ Tumour Profiling Unit, The Institute of Cancer Research, London SW3 6JB, UK. ${ }^{7}$ Center for Cancer Research, National Cancer Institute, Bethesda, MD 20892, USA. ${ }^{8}$ Wellcome Trust Sanger Institute, Hinxton, Cambridgeshire CB10 1SA, UK. ${ }^{9}$ UCSF Helen Diller Family Comprehensive Cancer Center, 1450 3rd St, San Francisco, CA 94158, USA. These authors contributed equally: Stephen J. Pettitt, Dragomir B. Krastev. Correspondence and requests for materials should be addressed to A.A. (email: Alan.Ashworth@ucsf.edu) or to C.J.L. (email: Chris.Lord@icr.ac.uk)
} 
D rugs targeting the poly-(ADP-ribose) polymerase (PARP) enzymes PARP1 and PARP2 cause synthetic lethality in tumour cells with homologous recombination (HR) defects, including those with loss-of-function mutations in the $B R C A 1$ or $B R C A 2$ tumour suppressor genes ${ }^{1-3}$. PARP1 acts as a DNA damage sensor, rapidly binding single- and double-stranded DNA breaks as they occur and then coordinating their repair by synthesising poly-(ADP-ribose) (PAR) chains on target proteins (PARylation) ${ }^{4}$. The rationale for using PARP inhibitors (PARPi) to treat HR-deficient cancers is based on the exquisite sensitivity of BRCA1- or BRCA2-defective cells to small-molecule PARPi, as well as the ability of Parp1 gene silencing to selectively inhibit Brca1- or Brca2-defective cells ${ }^{1,2}$. Subsequent experiments have revealed that in addition to inhibiting the catalytic activity of PARP1, most clinical PARPi cause cytotoxicity by trapping PARP1 at sites of DNA damage ${ }^{5-7}$, consistent with earlier observations that PARP1 displays a greater affinity for DNA breaks in the presence of toolbox PARPi ${ }^{8,9}$. The PARP1 trapping potency of different inhibitors correlates with their cytotoxic potency, with talazoparib (BMN673, Pfizer) showing the greatest effect ${ }^{5,6}$. Complete ablation of PARP1 expression by transposon-mediated mutagenesis or gene silencing in BRCA1/ BRCA2 wild-type (WT) cells results in extreme resistance to several PARPi $1,5-7$.

The ability of some PARPi to trap PARP1 might be partially explained by the observation that PARP1 DNA binding is independent of its catalytic activity, while dissociation of PARP1 from DNA requires PARylation ${ }^{10}$. Recent structural studies have proposed a model of PARP1 binding to singlestranded DNA damage that takes into account a series of molecular interactions between different PARP1 protein domains ${ }^{11-13}$. In its non-DNA-bound state, a regulatory PARP1 helical domain (HD) is proposed to prevent catalytic activity. Upon PARP1 DNA binding (via N-terminal zinc-finger (ZnF) DNA-binding domains), an unfolding of the PARP1 helical region accompanies catalytic activation and PARP1 synthesises PAR chains on itself and other acceptor proteins in the vicinity ${ }^{11-13}$. These PARylation events recruit other DNA repair enzymes, such as XRCC1 ${ }^{14}$, and act as a second messenger signalling the presence of DNA damage. The synthesis of highly negatively charged PAR chains on PARP1 is thought to also cause dissociation of PARP1 from DNA, presumably through a steric mechanism ${ }^{10}$.

Here we used CRISPR-Cas9 mutagenesis to investigate the mechanisms of PARPi toxicity in greater detail. We apply a focused mutagenesis approach to generate a large number of PARP1 mutant alleles that cause resistance, identifying an axis of intramolecular communication in PARP1 that mediates PARPi toxicity. We isolate PARP1 mutants from tumour cells with BRCA 1 exon 11 mutations and demonstrate that residual BRCA1 function in these cells allows tolerance of PARP1 loss of function, despite the synthetic lethal relationship between these genes. A PARP1 mutation observed in a tumour from a PARPi-resistant patient prevents PARP1 trapping, suggesting that PARP1 mutations that impair trapping could contribute to clinical PARPi resistance. Finally, we find that PARP1 mutations caused a distinct set of drug sensitivities when compared to other known forms of PARPi resistance (loss of REV7 (MAD2L2) or TP53BP1, or $B R C A 1$ reversion mutants), suggesting that knowledge of the molecular mechanism of resistance in individual patients could inform decisions on further treatment.

\section{Results}

In-frame Parp1 deletions cause PARPi resistance. Although PARPi are showing considerable promise as the first of a new generation of synthetic lethal therapies, resistance is a major issue $^{15,16}$. To better understand this, we carried out a genomewide CRISPR-Cas9 mutagenesis screen (encompassing 87 897 single guide RNAs (sgRNAs)) to identify mouse embryonic stem (ES) cell mutants resistant to the potent PARPi talazoparib $^{5,17}$ (BMN673). We isolated and analysed 24 resistant clones (Methods, Fig. 1a). Nine clones harboured one of two different sgRNAs targeting Parp1 (Table 1). Parp1 was the only gene that was targeted by more than one different sgRNA among the resistant clones (Table 1 and Fig. 1b).

Parp1 protein was absent in all of the PARPi-resistant clones with a Parp1 sgRNA (Fig. 1c) with one exception (clone BR8), consistent with the observation that ablation of PARP1 expression prevents PARP1 trapping and causes PARPi resistance ${ }^{5,7}$. DNA sequencing of the Parp1 target site in clone BR8 revealed two in-frame Parp1 mutations: c.127_129delATG and c. [127_129delATG;130T>A] (p.43delM and p.[43delM;44F>I], Fig. 1d). Both M43 and F44 residues are conserved between human and mouse PARP1 proteins and are predicted to be involved in base stacking interactions formed at the site of DNA/ PARP1 interaction by the ZnFs of the PARP1 DNA-binding domain ${ }^{18}$. Using the PARP1 trapping assay ${ }^{6}$, we found that mutant Parp1 protein was not associated with the chromatin (C) fraction after talazoparib treatment, in contrast to the WT protein (Fig. 1e), suggesting that Parp1 trapping was impaired. A PARP1GFP fusion protein with the p.[43delM;44F $>$ I] mutation also failed to be recruited to DNA damage produced by laser microirradiation and did not produce PAR at the irradiated site, as monitored by expression of a fluorescent PAR-binding reporter, PBZ-mRuby2 (Fig. 1f; Methods; and D.B.K. and C.J. L., manuscript submitted). The magnitude of talazoparib resistance in the BR8 mutant clone was similar to that seen in Parp1 mutants that showed complete loss of Parp1 protein expression, such as BR13 (Fig. 1g). The BR8 clone also exhibited resistance to olaparib (Fig. $1 \mathrm{~h}, p<0.0001$, analysis of variance (ANOVA)), suggesting a drug class effect. We also noted that Parp1 mutant ES cells exhibited enhanced sensitivity to camptothecin, a topoisomerase I inhibitor (Supplementary Figure 1a), consistent with previous observations ${ }^{19}, 20$. Taken together, these observations suggested that loss of Parp1 DNA binding and activity caused by mutations in the $\mathrm{ZnF}$ domain of the protein can drive PARPi resistance.

Parp1-independent resistance to talazoparib. We sequenced the Parp1 sgRNA target sites in all PARPi-resistant clones but only identified Parp1 mutations in clones with a Parp1 sgRNA. We therefore considered alternative explanations for PARPi resistance in the other clones identified from the screen. Although Parp1 was the only gene represented by multiple distinct sgRNAs in the PARPi-resistant cells, the hallmark of a true positive screen hit, we did identify multiple clones that carried either a sgRNA targeting $T d g$ or a sgRNA targeting $\operatorname{Trp53}$ (clones BR11, 14 and 24 for $T d g$ and BR5 and 21 for Trp53; Table 1). Tdg, which encodes thymine DNA glycosylase, was unusual in that there was only a single, rather than multiple, sgRNA design present in the screening library. We therefore designed an additional sgRNA (Supplementary Figure 1b) and generated independent $\mathrm{Tdg}$ defective clones, each of which confirmed the PARPi resistance phenotype associated with Tdg loss (Supplementary Figure 1c, d). Tdg mutants exhibited WT sensitivity to camptothecin or ionising radiation (Supplementary Figure 1a, e), and displayed WT Parp1 trapping characteristics in methyl methanesulfonate (MMS)treated cells (Supplementary Figure 1f), and thus did not phenocopy Parp1 mutants. Tdg removes thymine, as well as some modified uracil and cytosine, residues from DNA, thus creating 
abasic sites ${ }^{21}$. It seems possible that Tdg acts upstream of Parp1 to generate substrates for Parp1 trapping, and that in its absence, a reduction in Parp1 trapping causes drug resistance, a hypothesis previously suggested ${ }^{22}$. However, we were unable to detect a profound general defect in Parp1 trapping in Tdg mutants (Supplementary Figure 1f) suggesting that this phenotype might be more mechanistically complex or undetectable using this approach.

Further investigation of clones with guides targeting Trp53 (encoding the mouse homologue of p53) revealed that each had the same compound frameshift mutations and were thus likely sister clones (Supplementary Figure 1g). Sequencing of the ES cell

a

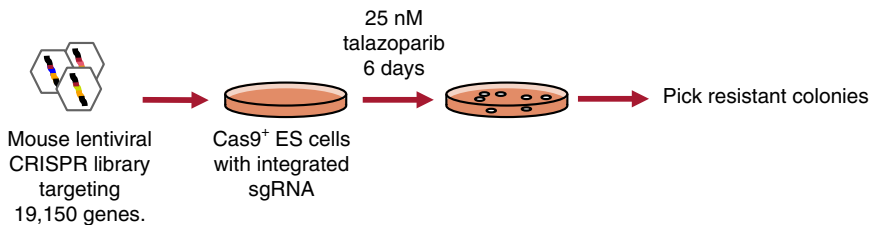

Extract genomic DNA

PCR amplify gRNA sequence

CRISPR library with integrate

19,150 genes.

b NCBIM38 chr $1180.57 \mathrm{Mb}$

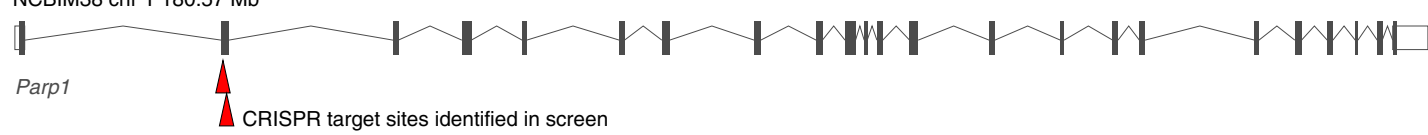

C

$$
\begin{array}{ll}
\text { sgRnA: } & \text { Parp1\#1 } \\
& \text { P Parp1 \#2 }
\end{array}
$$
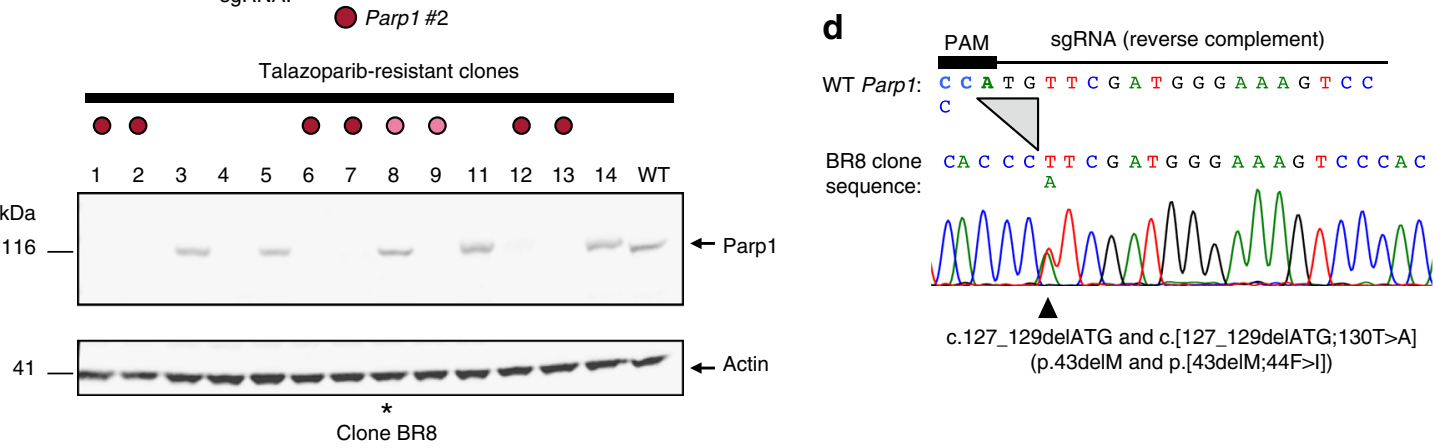

e

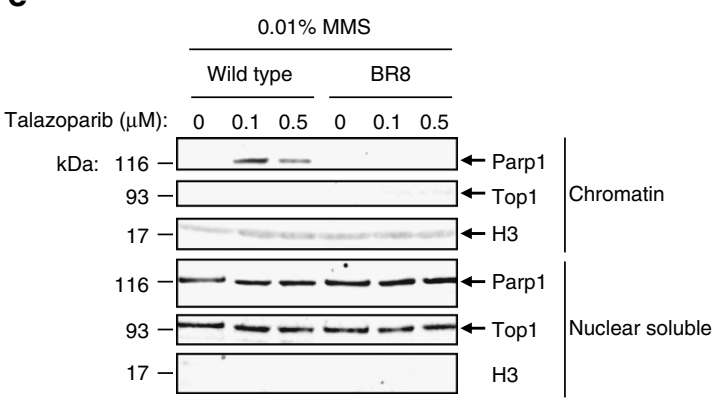

$\mathbf{f}$
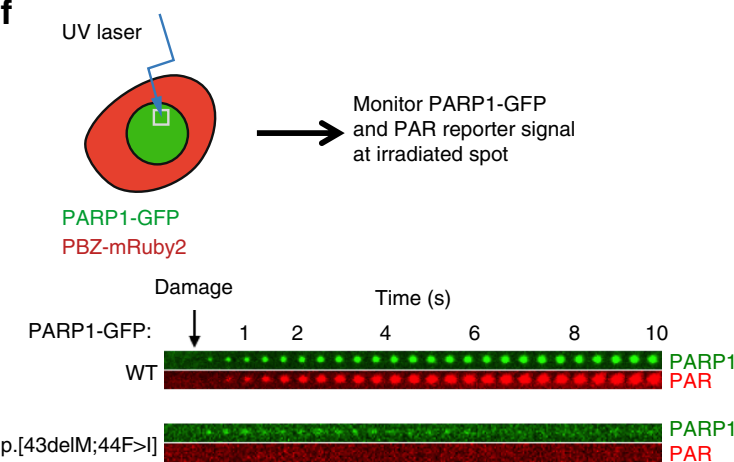

g

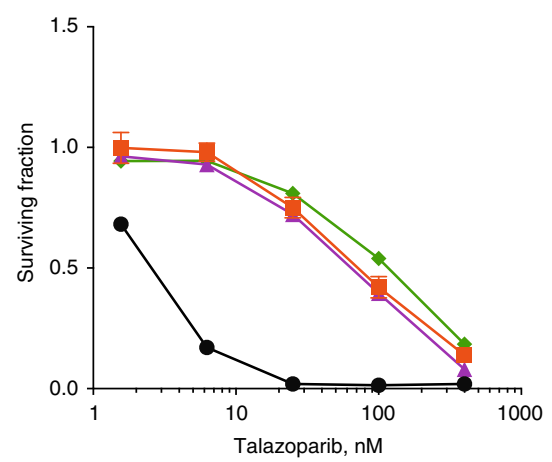

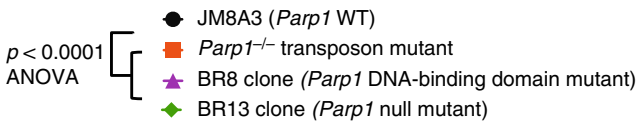

h

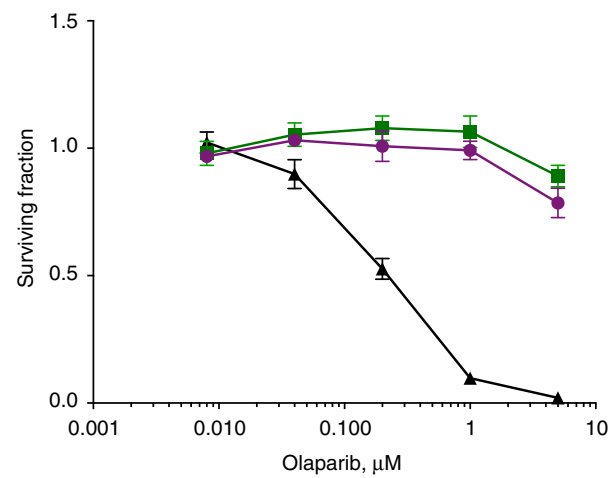

\footnotetext{
$p<0.0001\left[\begin{array}{l}- \text { BR8 clone (Parp1 DNA-binding domain mutant) } \\ - \text { BR13 clone (Parp1 null mutant) }\end{array}\right.$

ANOVA $L \neq \mathrm{JM} 8 \mathrm{~A} 3$ (Parp1 WT)
} 
library prior to selection revealed that all Trp53-targeting guides were highly enriched in the pre-PARPi-exposed population of cells (Supplementary Figure $1 \mathrm{~h}$ ). Although these clones did show significant talazoparib resistance (Supplementary Figure 1i, $p<$ 0.0001 , ANOVA), we would expect to have isolated more than one $\operatorname{Trp53}$ mutation event if this were a specific effect. It is therefore likely that these clones were isolated due to their high abundance in the pre-PARPi-exposed population.

\section{A focused CRISPR-Cas9 screen for in-frame PARP1 mutations.} Our CRISPR screens highlighted a key feature of CRISPR mutagenesis-the ability to cause and easily identify subtle mutations as well as null mutations, such as the p.43delM mutation in the BR8 clone (Fig. 1d). To study such mutations in more detail and to identify which regions of PARP1 are required for PARPi cytotoxicity, we designed an experimental approach to directly select PARPi-resistant cells with CRISPR-Cas9-induced mutations that preserved the native PARP1 reading frame (e.g., in-frame insertion/deletion mutations, rather than frameshift mutations that may cause production of truncated protein). This approach, which we term "tag-mutate-enrich" (Fig. 2a), used HeLa cells with a BAC transgene containing PARP1 gene fused to green fluorescent protein (GFP) coding sequence (encoding a PARP1 protein with a C-terminal GFP fusion). The GFP tag allowed us to enrich for cells with in-frame PARP1 protein expression by fluorescence-activated cell sorting (FACS) isolating the GFP-positive fraction. PARP1-GFP cells were mutated with a focused sgRNA library of 29 guide RNAs targeting PARP1 (Supplementary Data 1); PARPi-resistant cells were then selected via talazoparib exposure and the GFP-positive fraction isolated (Fig. 2a). The sgRNA library was introduced as six different lentiviral pools, grouped by reverse transcription-PCR (RT-PCR) product later used for genotyping. These products were amplified from GFP cDNA and sequenced using the Ion Torrent PGM platform (Fig. 2a). For all but one sgRNA pool we saw a significant enrichment of in-frame mutations in PARP1 in the PARPi-resistant population (compared to a null hypothesis of $1 / 3$ in-frame mutations; pools $1,2,4,5$ and $6: p<10^{-15}$, binomial test with alternative hypothesis $p$ (success) $>1 / 3,95 \%$ confidence interval (CI) 0.62-1.00. Pool 3, 95\% CI 0.24-1.00, $p=1$; Supplementary Figure 2a). Most of these in-frame mutations were close to a CRISPR target site (95\% of indels, represented by $70 \%$ of total reads, within $5 \mathrm{bp}$ of a predicted target site, $p<1 \times 10^{-5}$, based on Monte Carlo simulation of a randomly occurring mutation, Supplementary Figure 2b). By translating and aligning in-frame reads from PGM sequencing, we identified candidate PARP1 amino-acid residues associated with resistance. For example, most reads from the PARPi-resistant population generated from sgRNA pool 1 exhibited deletion of nucleotides encoding amino-acid residues K119 and S120 (Fig. 2b and Supplementary Figure 2a), which are both DNA-contacting residues within the second $\mathrm{ZnF}$ domain of PARP $1^{18}$. We also observed numerous other alleles with deletions or insertions affecting this region (Fig. 2b and Supplementary Data 2 and 3). Our subsequent microirradiation assay analysis demonstrated that a p.119_120delKS mutation abolished PARP1-GFP recruitment to the sites of DNA damage (Fig. 2c), suggesting that mutations in residues K119 and S120 might cause PARPi resistance by altering PARP1 DNA-binding properties.

Mutations outside the $\mathrm{ZnF}$ domains cause PARPi resistance. The focused sgRNA screen also identified three PARP1 mutations in residues not known to be directly involved in DNA binding: p.329N>Q (N329Q); p.742-743HD>F (HD742F); and p.848delY (848delY, Fig. 2d and Supplementary Data 2 and 3). N329 sits within the third $\mathrm{ZnF}$ of PARP1, but is not predicted to form part of the DNA-binding interface. HD742F is located within the HD, a regulatory region shown to be important for PARP activation $^{11,12}$. Y848 is part of a solvent-exposed helix of the catalytic domain (Fig. 2d). In cell-free assays using recombinant PARP1 proteins, we found the talazoparib $\mathrm{IC}_{50}$ to be similar for mutant and WT PARP1 proteins (Supplementary Figure 2c, d), suggesting that the PARPi resistance phenotypes were not caused by differences in the ability of PARPi to inhibit the catalytic activity of mutant proteins. PARP1 trapping assays in CAL51 $P A R P 1^{-1-}$ cells transfected with mutant or WT PARP1-GFP cDNA expression constructs suggested that N329Q and HD742F mutant proteins were not trapped by PARPi (Fig. 2e), explaining the PARPi-resistant phenotypes associated with these mutations. In contrast, PARP1-848delY-GFP was trapped in the chromatin fraction by talazoparib, but to a lesser extent than WT PARP1GFP (Fig. 2e). Laser microirradiation assays confirmed this partial PARP1 trapping phenotype with the PARP1-848delY-GFP protein; whilst WT PARP1-GFP was rapidly recruited to the site of microirradiation (peak $\approx 4 \mathrm{~s}$ ) and induced PAR formation in a similar time frame (Fig. 2f), the extent of PARP1-848delY-GFP recruitment to the site of microirradiation was reduced, as was PARylation (Fig. 2f). As a negative control, PARP1-N329Q-GFP exhibited limited recruitment to damaged DNA (Fig. 2f). The addition of talazoparib delayed PARP1-848delY-GFP dissociation from microirradiated sites, but to a lesser extent than for WT PARP1-GFP (Supplementary Figure 2e, f, $p=5 \times 10^{-7}, t$-test). PARP1-848delY-GFP also dissociated faster from microirradiated sites, being absent from microirradiated regions $30 \mathrm{~min}$ after microirradiation; WT PARP1-GFP still showed $40 \%$ of maximal trapping at this timepoint (Supplementary Figure 2f,

\footnotetext{
Fig. 1 A genome-wide CRISPR screen for PARP inhibitor resistance identifies in-frame Parp1 mutants. a Experimental scheme. b Locations of Parp1 guide RNA target sites in exon two of the mouse Parp1 gene. c Parp1 western blot of lysates from talazoparib-resistant clones identified in the CRISPR screen. Individual clones are colour-coded according to sgRNA present (see key). Clones 1, 2, 6, 7, 9, 12 and 13 with Parp1 sgRNAs have lost Parp1 protein expression, whilst Parp1 sgRNA clone $8\left(B R 8,{ }^{\star}\right)$ has retained Parp1 expression. d Clone BR8 has an in-frame Parp1 deletion and a Parp1 substitution mutation. Sanger sequencing trace of the Parp1 sgRNA target site is shown, illustrating a 3 bp deletion on both alleles and a heterozygous c.130T>A substitution mutation ( $p .44 \mathrm{~F}>1$ ) close to the CRISPR PAM site. e Parp1 is not trapped in the chromatin fraction by PARP inhibitor in the BR8 clone. Western blots illustrating Parp1 in the chromatin and nuclear soluble fractions of wild-type ES cells and Parp1 mutant BR8 cells exposed to talazoparib. Data shown are representative of two experiments. $\mathbf{P A R P 1}$ protein with a $\mathrm{p} .[43 \mathrm{delM} ; 44 \mathrm{~F}>\mathrm{I}]$ mutation has impaired recruitment to damaged DNA and does not initiate PAR synthesis at damaged DNA. Localisation of PARP1-GFP to damaged DNA was estimated by visualising GFP signal at the microirradiated spot, as was the generation of PAR at the damaged site by use of a PAR-binding PBZ-mRuby2 probe (see schematic). The time course of PARP1-GFP and PBZ-mRuby2 signals from CAL51 PARP1 ${ }^{-1}$ - cells transfected with PARP1-GFP (top) and PARP1-p.[43delM;44F>I]-GFP (bottom) are shown. "Damage" denotes time at which microirradiation was carried out. $\mathbf{g}, \mathbf{h}$ Dose response curves illustrating that clone BR8 is resistant to both talazoparib ( $\mathbf{g}$ ) and olaparib (h). BR13 is an ES cell mutant with no Parp1 protein expression (see panel c); a transposon-mutagenised, Parp1 null mutant ${ }^{7}$ is also shown (red). Clone BR8 vs. BR13 and Parp $^{1 /-}$ transposon, $p=\mathrm{ns}$, ANOVA. Parp1 mutant clones vs. wild-type cells, $p<0.0001$, ANOVA. Mean of five replicates plotted, error bars show SD. Surviving fractions were calculated relative to DMSO-exposed cells for each mutant
} 


\begin{tabular}{|c|c|c|c|}
\hline Clone & $\begin{array}{l}\text { Likely cause of } \\
\text { resistance }\end{array}$ & Clonal with & sgRNAs identified \\
\hline BR1 & Parp1 & 7 & Parp1 (\#1), Tmed5 \\
\hline BR2 & Parp1 & - & Parp1 (\#1) \\
\hline BR3 & & - & Gpd2 \\
\hline BR4 & & - & $\begin{array}{l}\text { Tbx20, Gpr89, Pde4d, } \\
\text { Arhgap12 }\end{array}$ \\
\hline BR5 & & 21 & Trp53, Csmd1 \\
\hline BR6 & Parp1 & - & Parp1 (\#2), Apol6 \\
\hline BR7 & Parp1 & 12,1 & $\begin{array}{l}\text { Parp1 (\#1), Abca14, Dtnb, } \\
\text { Tmed5 }\end{array}$ \\
\hline BR8 & Parp1 \#2 & - & Parp1 (\#2), Lemd1, SImo1 \\
\hline BR9 & Parp1 \#2 & - & Parp1 (\#2) \\
\hline BR10 & Parp1 & - & $\begin{array}{l}\text { Parp1 (\#1), Spock1, P2ry6, } \\
\text { AW209491 }\end{array}$ \\
\hline BR11 & $T d g$ & 14 & Tdg, Gm5597 \\
\hline BR12 & Parp1 (by clonality) & 7 & Abca14, Dtnb \\
\hline BR13 & Parp1 & - & Parp1 (\#1), Nwd1, Scn10a \\
\hline BR14 & $T d g$ & 11 & Tdg, Gm5597, Tmed5 \\
\hline BR15 & & - & ND \\
\hline BR16 & & - & Defb25 \\
\hline BR17 & & - & ND \\
\hline BR18 & & - & Traf3ip1, Hkdc1, Gm4876 \\
\hline BR19 & & - & $\begin{array}{l}\text { Dnajb11, Scaf8, Acbd3, } \\
\text { Msh2 }\end{array}$ \\
\hline BR2O & & - & ND \\
\hline BR21 & & 5 & Trp53, Csmd3, Chst15 \\
\hline BR22 & & - & Cyp2r1, Tmem69 \\
\hline BR23 & & - & ND \\
\hline BR24 & $T d g$ & - & $T d g$ \\
\hline
\end{tabular}

Where clonality can be inferred from the combination of different guides observed, this is shown in column three

ND not determined (failure of PCR and/or sequencing)

$p=5.5 \times 10^{-3}, t$-test). These data therefore suggested that even the relatively subtle defect in trapping in the $848 \mathrm{delY}$ mutant (Fig. 2e, f) might be sufficient to cause PARPi resistance.

We mapped the residues affected by the mutations that we observed onto a previously described crystal structure of PARP1$\mathrm{ZnF}$ domains 1 and 2 together with the WGR, regulatory and catalytic domains ${ }^{13}$. We found that several of the PARPi resistance-causing mutations affected amino-acid residues (D45, H742, D743 and E688) involved in hydrogen-bonding interactions that bridge the DNA-binding domain and the catalytic domain (Fig. 2g), suggesting that these might control interdomain interactions that mediate PARP1 trapping; similar interdomain domain interactions link DNA binding to activation of PARP1 catalytic activity ${ }^{11-13}$. Taken together, these data established that mutations outside of the DNA-binding domain can cause PARPi resistance, likely by impairing PARP1 trapping.

PARP1 mutations cause PARPi resistance in BRCA1 mutant cells. We next assessed whether PARP1 mutations could cause PARPi resistance in a clinically relevant setting, such as in BRCA1 mutant tumour cells. Complete loss of both PARP1 and BRCA1 is expected to be synthetic lethal ${ }^{1,2}$. However, a growing body of evidence suggests that many pathogenic BRCA1 mutations may not result in complete loss of function. For example, SUM149 (also known as SUM149PT) triple-negative breast tumour cells possess a commonly occurring hypomorphic BRCA1 exon 11 c.2288delT frameshift mutation and loss of the WT BRCA1 allele ${ }^{23}$. As a consequence, SUM149 cells do not express the full- length BRCA1 p220 protein, but do express a hypomorphic splice variant of BRCA1, $\Delta 11 \mathrm{~b}$, which excludes the c.2288delT premature truncating mutation (along with most of the exon 11 coding sequence), but has some residual function ${ }^{24,25}$. We and others have previously confirmed that the BRCA1 mutation in SUM149 cells causes sensitivity to PARPi by demonstrating that genetic reversion of the BRCA1 mutation in SUM149 cells via CRISPR-Cas9 mutagenesis imparts PARPi resistance ${ }^{25,26 .}$

We carried out a genome-wide PARPi resistance CRISPR-Cas9 screen in SUM149 cells, using a previously validated sgRNA library $^{27}$, similar to our earlier screen in mouse ES cells. Out of 12 talazoparib-resistant clones analysed, 8 possessed one of three PARP1 sgRNAs (Table 2), suggesting that talazoparib cytotoxicity is mediated by PARP1 even in this BRCA1 mutant cell line. To confirm this observation, we infected Cas9-expressing SUM149 cells with a lentiviral sgRNA vector targeting the PARP1 coding sequence homologous to the p.43/44 mutation site in the PARPiresistant mouse ES cell BR8 clone we identified earlier (Fig. 1d). This sgRNA induced talazoparib resistance (Fig. 3a). We subcloned two daughter clones from the PARPi-resistant SUM149 population, TR1 and TR2; TR1 had four different PARP1 alleles with frameshift mutations and lacked PARP1 protein expression, whilst TR2 expressed apparently full-length PARP1 protein (Fig. 3b and Supplementary Data 4). TR2 possessed three different PARP1 alleles with frameshift mutations (Table 3 and Supplementary Data 4) but also a p.43_45delMFD in-frame deletion, similar to the p.43/44 mutations identified in PARPi-resistant BR8 mouse ES cells (Fig. 1d). This supported the hypothesis that the p.43delM mutation identified in the mouse BR8 clone (Fig. 1d) was likely to be the cause of PARPi resistance, as opposed to a passenger effect that co-occurred in a clone with a non-PARP1-mediated mechanism of resistance. No PARP1 WT DNA-sequencing reads were identified among subcloned PARP1 PCR products from either TR1 or TR2 (Supplementary Data 4). The allele frequencies of the PARP1 mutations in TR1 and TR2 suggested that there were five copies of the PARP1 locus in SUM149 cells, confirmed by single-nucleotide polymorphism genotyping of SUM149 (Supplementary Data 4; A. Grigoriadis, personal communication). Both clones remained talazoparibresistant after culture in the absence of PARPi, demonstrating that this was a stably acquired phenotype (Fig. 3c, ANOVA, $p<$ 0.0001). We did not find evidence for other known mechanisms of PARPi resistance in TR1 and TR2, such as reversion of fulllength BRCA1 expression ${ }^{28}$ (Supplementary Figure 3a), 53BP1 or REV7 (MAD2L2) $\operatorname{loss}^{29}$ (Supplementary Figure 3b, c), or an increase in DNA damage-induced nuclear RAD51 foci (Supplementary Figure 3d). This suggested that PARP1 mutation or loss could not only be tolerated in SUM149 cells but also caused PARPi resistance.

By generating a series of isogenic SUM149 daughter clones with different mechanisms of PARPi resistance (PARP1 mutation, BRCA1 reversion, deleterious TP53BP1 or REV7 (MAD2L2) mutation $^{28-32}$; Supplementary Figure $4 \mathrm{a}$ ), we also found that SUM149 cells with PARP1 mutations exhibited a comparable, if not greater, level of PARPi resistance than previously identified mechanisms of resistance (Supplementary Figure 4b, c; $p<$ 0.0001, ANOVA compared to parental cells for all mutants). PARP1 mutant SUM149 cells had the most profound PARPiresistant phenotype seen amongst 38 molecularly diverse breast tumour cell lines (Supplementary Figure 5). We also found that PARP1 mutant SUM149 cells did not exhibit cross-resistance to cisplatin that might be expected if BRCA1 function had been restored in these cells ${ }^{26}$ (Supplementary Figure 6a); in comparison, 53BP1 mutant clones had an intermediate level of resistance to cisplatin $\left(p<0.0001\right.$, ANOVA), as previously described ${ }^{29}$, as did clones with a BRCA1 reversion, whilst $R E V 7$ mutants 
exhibited enhanced sensitivity to cisplatin $(p<0.0001$, ANOVA), a possible consequence of losing REV7's role in translesion synthesis $^{33}$ (Supplementary Figure 6a). PARP1 mutation in SUM149 cells enhanced the existing topoisomerase I inhibitor sensitivity caused by BRCA1 mutation (Supplementary Figure 6b-d, $p<0.0001$, ANOVA), consistent with the enhanced camptothecin sensitivity caused by Parp1 mutation in mouse ES cells (Supplementary Figure 1a) and previous observations 19,20 . These observations suggested that PARP1 mutation in BRCA1 mutant tumour cells caused a similar extent of PARPi resistance as for known mechanisms of resistance, but had differing effects on platinum sensitivity.

To assess whether the PARPi-resistant phenotype caused by PARP1 mutation might also operate in vivo, we injected SUM149-Cas9 and PARP1 mutant SUM149-TR2 cells subcutaneously in $\mathrm{BALB} / \mathrm{c}$ nude mice. Once tumours had established, we randomised mice into one of two treatment cohorts, one treated with the clinical PARPi talazoparib and the other with the drug

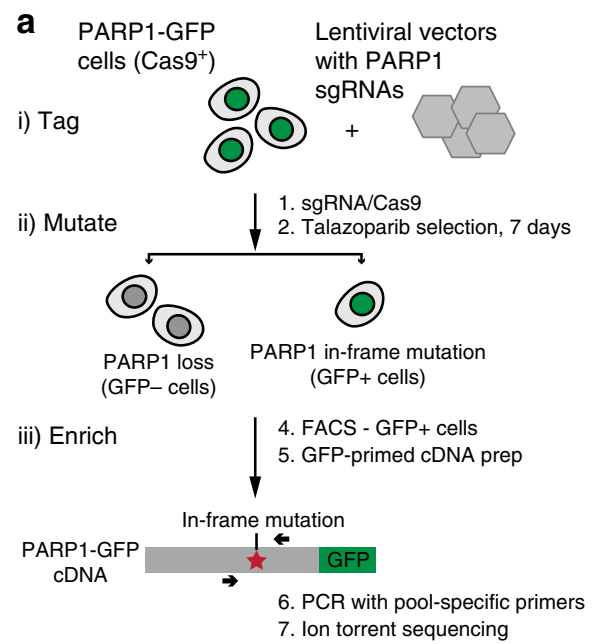

\section{b}

Talazoparib-resistant PARP1 mutants

PARP1 WT $1 \wedge^{9} \imath^{1}$

TLGDFAAEYAKSNRSTCKGCME TLGDFAAEYA--NRSTCKGCME TLGDFAAEYA--NRSTCKGCME TLGDFAAD-----RSTCKGCME TLGDFAADYA--NRSTCKGCME TLGDFAAEYA--NRSTCKGCME TLGDFAAEYA--NRSTCKGCME TLGDFAAEYAK---CTCKGCME TLGDFAAEYAKY-RSTCKGCME TLGDFAAEYAKS-RSTCKGCME TLGDFAAEYATN-RSTCKGCME TLGDFAAEY-------ACKGCME
C

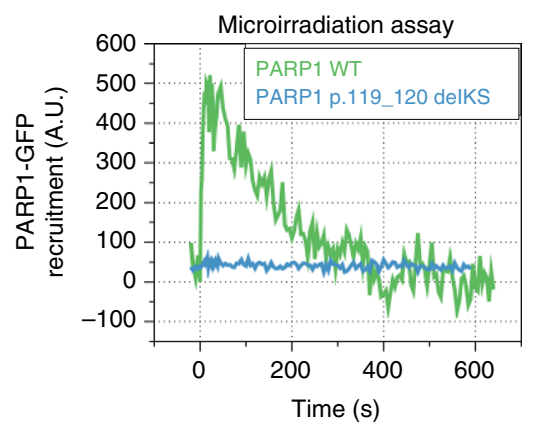

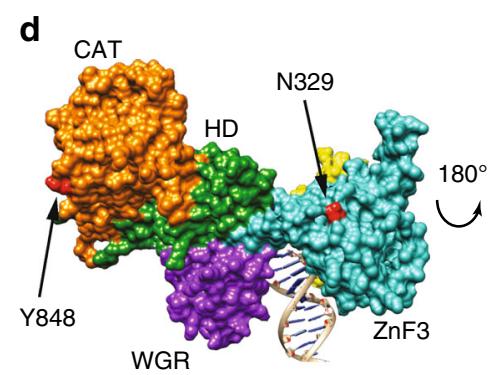
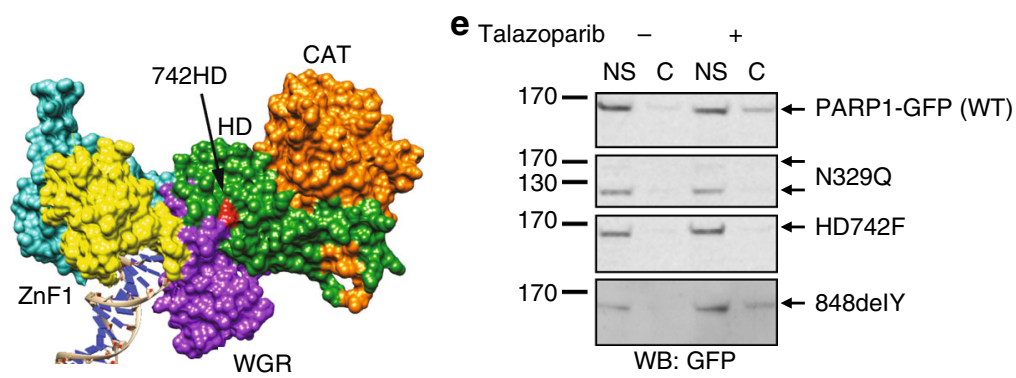

f
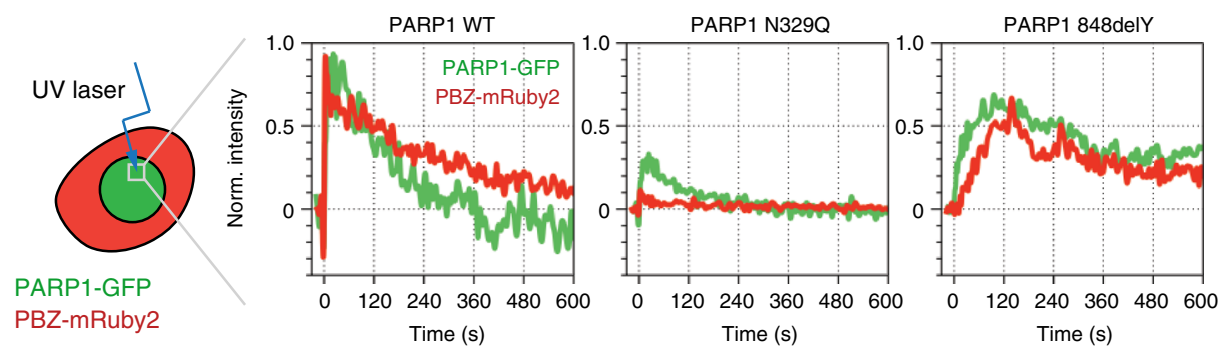

g

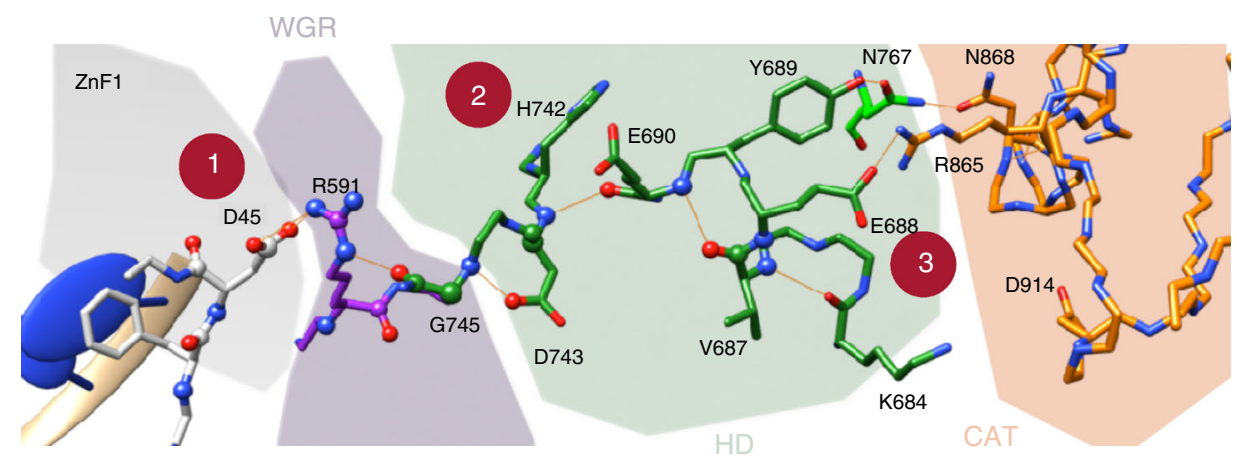


vehicle alone. Whilst talazoparib treatment delayed the growth of SUM149-Cas9 xenografts and extended the survival of xenograftbearing mice, compared to vehicle treatment $(p=0.0086$, logrank test, Fig. 3d), it did not have any detectable effect in mice bearing PARP1 mutant SUM149-TR2 xenografts $(p=0.98$, logrank test).

Residual BRCA1 function supports PARP1 loss in tumour cells. We also found that PARP1 sgRNA caused PARPi resistance in BRCA1 mutant COV362 ovarian tumour cells ${ }^{34}$ (BRCA1 c.2611fs [exon 11] and c.4095+1G>T [exon 11 splice donor]), suggesting that these observations were not private to SUM149 cells. COV362 cells were infected with six different pools of lentiviral PARP1 sgRNAs as used in the HeLa screen above (Fig. 2) and selected in talazoparib. We found profound talazoparib resistance in all sgRNA lentivirus-infected populations, except those infected with PARP1 sgRNA pool six, where resistance was less-pronounced, although still significant (Fig. 3e; $p<0.0001$ in each case, ANOVA compared to parental COV362-Cas9 cells). We also isolated a number of daughter clones from the PARPiresistant COV362 populations. Some of these clones had lost PARP1 expression while others had in-frame indels in PARP1, including a clone with a p.119delKS mutation and three independently derived clones all with p.848delY/p.848_849delYK compound mutations (referred to as p.848delY/YK, Supplementary Figure $7 \mathrm{a}$ and Table 3). All clones with PARP1 mutations remained resistant to talazoparib after culture without PARPi selection during subcloning and expansion (Supplementary Figure $7 \mathrm{~b}, \mathrm{c}$ ). Interestingly, COV362 clones isolated with Y848 mutations at the endogenous PARP1 locus (p.848delY/delYK) showed a less-pronounced resistance phenotype compared to complete null clones, although the extent of resistance was still significant compared to WT cells (Supplementary Figure 7b, c, d; $p<0.0001$, ANOVA). These clones also showed some residual PARylation activity in agreement with the microirradiation phenotype observed earlier (Supplementary Figure $7 \mathrm{e}$ and Fig. 2f), suggesting an intermediate level of PARPi resistance could be caused by a partial PARP1 trapping defect.

We also attempted to isolate PARP1 mutants from MDA-MB436 cells, which have a BRCA1 c.5396+1G $>$ A mutation in the splice donor site of exon 20. This mutation results in a truncated $\mathrm{BRCA1}$ protein lacking the BRCT region required for $\mathrm{HR}^{23}, 35$. We were unable to isolate long-term talazoparib-resistant cells from this cell line after transfection with PARP1 sgRNAexpressing lentiviral pools. Analysis of cells grown in talazoparib for a short period (Supplementary Figure $7 \mathrm{f}$ ) showed that mutations were induced at the sgRNA target sites, but cells with PARP1 mutations did not survive in the long term (Supplementary Figure 7g). In contrast, sequencing of COV362 cells infected with the same PARP1 sgRNA pools revealed that several pools of talazoparib-resistant cells generated in the same way had mutagenized all PARP1 alleles in all cells, indicated by very low or absent WT reads (Fig. 3e and Supplementary Figure 7g). This suggested that mutation of all PARP1 alleles was not tolerated in MDA-MB-436 cells, in contrast to SUM149 or COV362 cells.

We therefore considered it possible that the residual BRCA1 function in SUM149 and COV362 might allow these cells to tolerate PARP1 mutations. To address this, we inhibited the residual BRCA1 function in SUM149 and COV362 cells with siRNA targeting BRCA1 target sites outside exon 11; doing the same in SUM149/COV362 PARP1 mutants isolated earlier demonstrated that BRCA1 siRNA had a more profound cell inhibitory effect in PARP1 mutant COV362 and SUM149 cells, than in PARP1 WT parental cells (Fig. $3 \mathrm{f}-\mathrm{h}, p<0.05$ for all cases compared to parental cells, $t$-test). This suggested that some residual BRCA1 function might exist in these cells and is required for cell survival in the face of PARP1 mutation. BRCA2 siRNA also selectively targeted PARP1 mutant COV362 and SUM149 cells, suggesting some requirement for BRCA2 function in these cells despite the BRCA1 mutation (Fig. $3 \mathrm{f}-\mathrm{h}, p<0.05, t$-test). Taken together, these observations suggested that PARP1 loss might be tolerated in COV362 and SUM149 cells due to some residual BRCA1-dependent function. Exon 11 frameshift mutations comprise approximately $30 \%$ of pathogenic BRCA1 mutations identified to date ${ }^{25}, 36-39$ and thus PARP1 mutation and/or loss could be a clinically relevant mechanism of PARPi resistance in this specific context.

A dense CRISPR screen for functional annotation of PARP1. To investigate which functions of PARP1 were important for cytotoxicity of talazoparib in BRCA1 mutant cells, we designed a new tiling CRISPR library, comprising 489 guides, designed to give the densest possible coverage of PARP1 mutations

\section{Table 2 sgRNA sequences identified in talazoparib-resistant SUM149-Cas9 cells mutagenized with a genome-wide CRISPR library}

SgRNA Colonies

PARP1_ex16

PARP1_ex19

PARP1_ex22

PARP1_ex19, MCF2L_ex2

PARP1_ex22, JPH4_ex3

RABGAP1_ex8

ND ( $>2$ guides)

Fig. 2 A focused CRISPR-Cas9 mutagenesis "tag, mutate and enrich" screen identifies PARP1 mutations outside the DNA-binding domain that cause PARP inhibitor resistance. a Experimental "tag, mutate and enrich" scheme to isolate missense and in-frame PARP1 mutants associated with PARP inhibitor resistance. $\mathbf{b}$ Translated alignment of PARP1 amino-acid mutations identified in talazoparib-resistant cells isolated from lentiviral sgRNA pool 1 (designed to target $\mathrm{ZnF1}$ and ZnF2 domains) from the screen shown in a. By comparing the multiple different mutations isolated from pool 1, a PARP1 p.119_120delKS minimal mutation associated with resistance was identified. Some insertions and larger deletions are omitted for clarity (see Supplementary Data 2). c PARP1 p.119_120delKS mutation abolishes recruitment of a PARP1-GFP fusion to sites of microirradiated DNA damage. CAL51 PARP1/- cells were

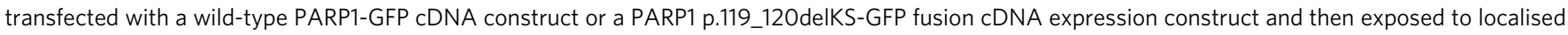
ionising radiation (a microirradiated spot) as in $\mathbf{f}$. Time course of PARP1-GFP signal at microirradiated site is shown. $\mathbf{d}$ Location of three mutations associated with PARPi resistance on a model of the PARP1 DNA structure ${ }^{12}$. e PARP1-N329Q and HD742F mutations ablate PARP1 trapping, while 848 delY

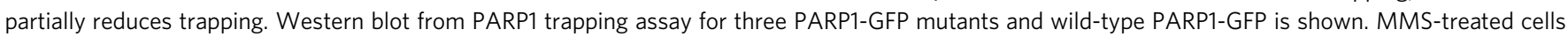

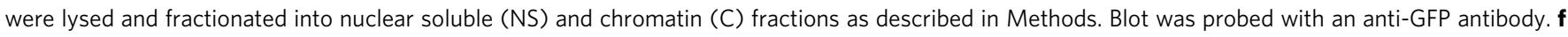
PARP1-848delY mutation alters PARP1 localisation kinetics at sites of DNA damage. Microirradiation and PAR synthesis phenotypes of N329Q and

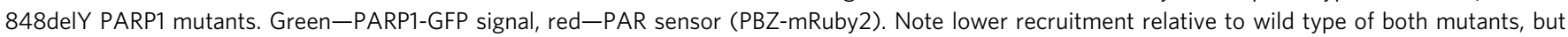
retention of PAR synthesis in 848delY. $\mathbf{g}$ Model of intramolecular communication between the DNA-binding ZnF1 domain and the catalytic (CAT) domain based on mutants identified from screens. (1) 45delD mutation in first zinc finger, (2) HD742F mutation shown in d, (3) E688 (Supplementary Data 2) 
(Supplementary Data 5 and Fig. 4a) and carried out a tiling screen in SUM149 cells in which the endogenous PARP1 locus had been tagged with a C-terminal GFP coding sequence using the CRISPaint system ${ }^{40}$. After mutagenesis followed by talazoparib selection and sorting GFP-positive cells as before, we sequenced the PARP1 coding sequence from GFP cDNA using a panel of 15 amplicons (Supplementary Data 6). This screen further illuminated the roles of different areas of PARP1 in PARPi toxicity. In agreement with our previous results, a large number of mutations were isolated which affected residues associated with either direct DNA binding (Fig. 4b, c; Supplementary Data 7) or the interfaces between $\mathrm{ZnF}$ domains (Fig. 4d). We also observed a high density of mutations associated with PARPi resistance affecting the WGR domain (Fig. $4 \mathrm{~b}, \mathrm{e}$ ). Based on our previous a

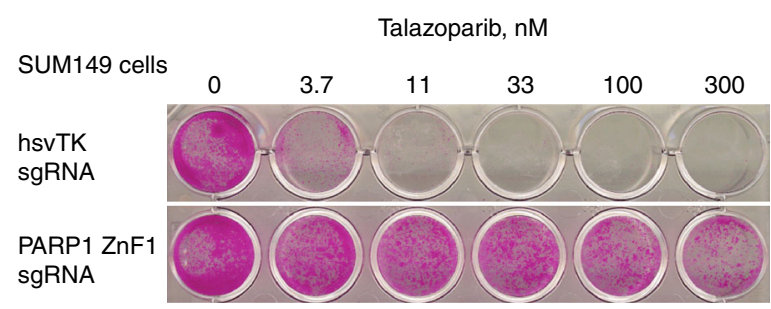

b

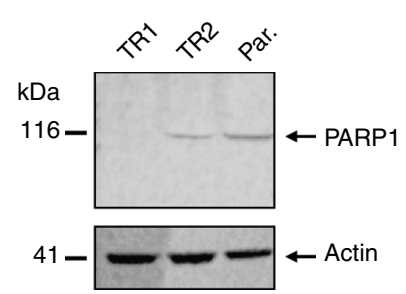

C

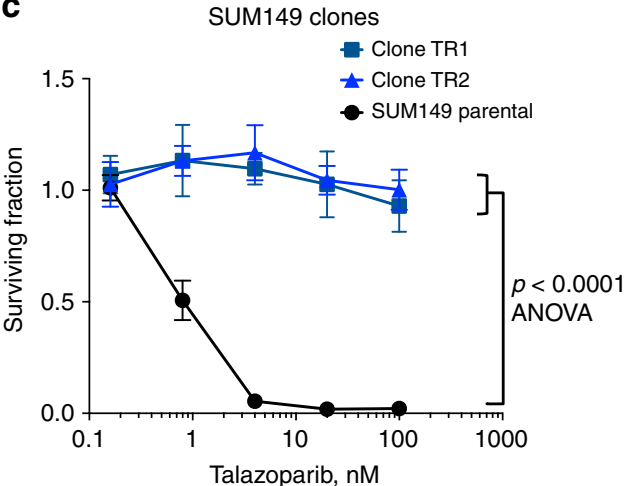

e

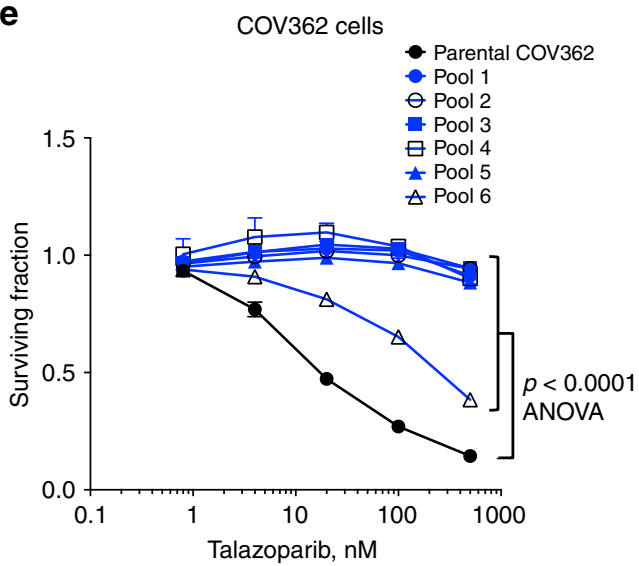

g

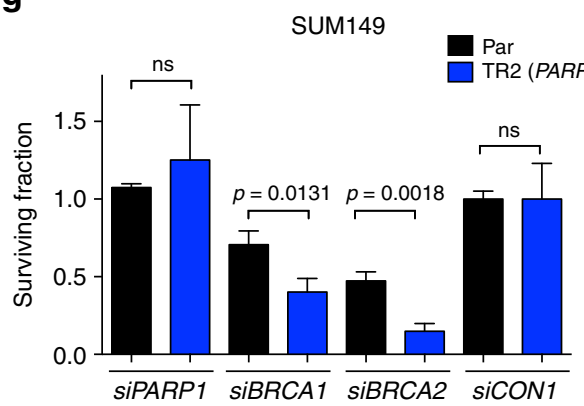

d

SUM149-TR2 (PARP1-1P.43МMFD)
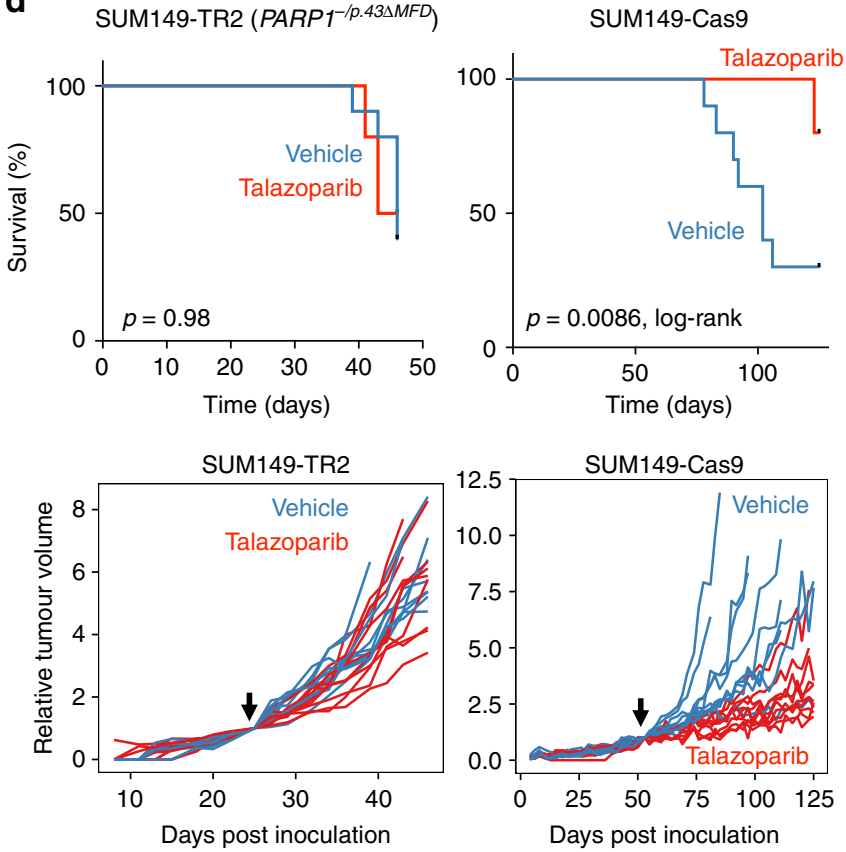

$\mathbf{f}$

SiRNA

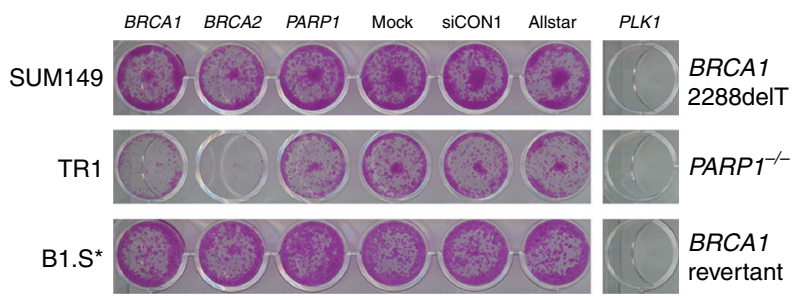

h

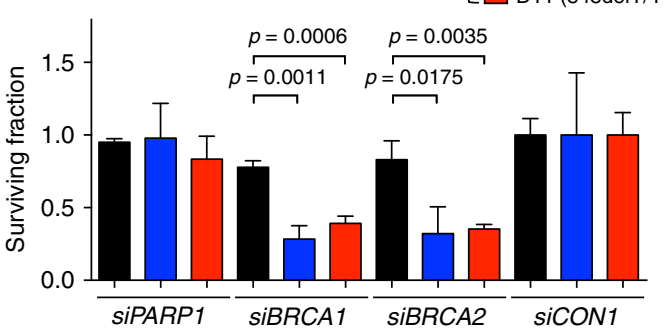


screen, we hypothesised that points of contact of the WGR domain with the $\mathrm{ZnF}$ and $\mathrm{HD}$ (regulatory) domains might be important for PARPi resistance and PARP1 function and activation (Fig. 2f); in this new screen we identified several mutation clusters at these points of contact (Fig. 4b, e; Supplementary Figure 8a) supporting this hypothesis. Although we identified few mutations in the catalytic domain that caused PARPi resistanceperhaps because mutations directly disrupting the catalytic activity would cause constitutive trapping and cytotoxicity-the most frequent mutation in the catalytic domain isolated in the screen affected residue A925, which is juxtaposed with residue Y848 in the three-dimensional structure of PARP1, further suggesting an important role for this region of the catalytic domain in PARPi cytotoxicity (Fig. 4e, inset 2). The relative lack of mutations in the BRCT domain, despite ample sequencing and sgRNA coverage in this area (Fig. 4c and Supplementary Figure $8 \mathrm{~b}$ ) might indicate that this region is dispensable for PARPi cytotoxicity and trapping.

PARP1 mutation observed in an olaparib-resistant patient. In parallel with these genetic studies, we identified a PARP1 p.R591C mutation $(c .1771 \mathrm{C}>\mathrm{T})$ in an ovarian cancer patient who showed de novo resistance to olaparib. The R591C mutation affects the WGR domain at the point of contact with D45 and the HD domain; our model (Fig. $2 \mathrm{~g}$ ) and previous work ${ }^{11-13}$ predict that this would be a critical residue for inter-domain communication in PARP1, between the DNA-binding and catalytic domains, implying that this mutant might bind DNA but would not show trapping. To investigate this, we assessed the recruitment of a PARP1-R591C-GFP fusion protein to sites of microirradiation in the presence and absence of the PARPi talazoparib. We found that whilst PARP1-R591C-GFP was recruited to sites of microirradiation, its dissociation from these sites was rapid compared to WT PARP1-GFP; furthermore, PARP1-R591C-GFP was not trapped on DNA by talazoparib, unlike WT PARP1-GFP (Fig. 4f).

\section{Discussion}

A key observation that contributed to the trapping hypothesis of PARPi cytotoxicity is that in HR proficient cells, PARP1 itself is required for cytotoxicty 6,7 . Our genome-wide CRISPR screen revealed that point mutations in the $\mathrm{ZnF}$ domains that abolish DNA binding were sufficient for this resistance, providing direct genetic evidence for the trapping hypothesis.

Our genetic screens also uncovered several clusters of mutations that suggest that regions of PARP1 outside the DNAbinding domain can influence trapping (Figs. 2d and 4b), observations that are consistent with inter-domain interactions being critical for PARP1 binding and activation ${ }^{1-13}$. These observations are also supported by an orthogonal approach using ethyl methyl sulfonate mutagenesis screens in haploid cells ${ }^{4}$. These observations add weight to the "reverse allostery" hypothesis proposed by Murai et al. ${ }^{6}$, which suggests that PARPi binding to the catalytic domain of PARP1 allosterically influences interactions between DNA and the N-terminal DNA-binding domains of the protein, to the extent that PARP1 becomes trapped on DNA. It is possible that the PARP1 mutations we have identified here are in amino-acid residues critical to this reverse allosteric process and therefore prevent allosteric enhancement of DNA binding upon inhibitor binding.

We also isolated a PARP1 mutant, p.848delY, which was associated with PARPi resistance despite exhibiting some residual PARP1 trapping. This reduced level of trapping might be sufficient to cause resistance-the amount of trapped PARP1 required to induce cytotoxicity is not known. Nor is it known whether all trapping, as observed by chromatin fractionation assays in the presence of alkylating agents, is equal with respect to cytotoxicity -PARP1 trapping at certain sites or lesion types may be more toxic than at others. It is possible that Tdg is a source of such lesions in ES cells, as loss of this specific glycosylase was sufficient for pronounced PARPi resistance (Supplementary Figure 1d). Alternatively, it is possible that PARP1 trapping is not, in itself, sufficient for cytotoxicity and other factors altered by the mutations are also required. For example, the Y848 residue is part of a solvent-exposed helix in PARP1 that may participate in protein-protein interactions-for example, with Timeless ${ }^{42}, 43$ that could be important for generating a DNA lesion that is cytotoxic. Mutations in another residue (A925) juxtaposed with Y848 in the PARP1 tertiary structure also led to PARPi resistance, possibly via a similar mechanism (Fig. 4e). It is possible that a larger trapped complex of PARP1 and interacting partner(s) is responsible for cytotoxicity, or that trapped PARP1 $1^{848 \mathrm{delY}}$ is altered in such a way as to not be cytotoxic. Nevertheless, the resistance phenotype of cells with mutations affecting Y848 at the endogenous PARP1 locus is slightly less-pronounced than a complete PARP1 knockout (Supplementary Figure 7d), supporting the hypothesis that the residual PARP1 trapping corresponds to some residual PARPi cytotoxicity. It will be interesting to study this region further to pinpoint the cause of the reduced cytotoxicity.

Our experiments also showed that PARP1 mutation can be tolerated in certain BRCA1 mutant, PARPi-sensitive tumour cells. This suggests that PARP1 trapping still underlies the increased cytotoxicity of PARPi in these tumour cells but that some residual BRCA1 function allows these cells to tolerate PARP1 mutations (Fig. 3f-h), consistent with previous observations that some

Fig. 3 PARP1 mediates PARPi sensitivity in BRCA1 mutant cell lines. a Cas9-expressing BRCA1 mutant SUM149 cells were transduced with PARP1-ZnF or hsvTK (non-targeting control) lentiviral sgRNA particles, selected in puromycin and exposed to the indicated talazoparib concentrations for 7 days. Representative SRB-stained 24-well plate image is shown. b Western blot showing PARP1 expression in talazoparib-resistant SUM149 clones TR1 and TR2. Par, parental SUM149 cells. TR2 has an in-frame PARP1-p.43_45deIMFD mutation. c SUM149 clones TR1 and TR2 are highly talazoparib-resistant. Plot shows survival relative to DMSO-exposed cells after exposure to the indicated concentration of talazoparib for 7 days. Mean of five replicates plotted, error bars show SD. d PARP1 mutant SUM149-TR2 xenografts do not respond to talazoparib treatment, whereas PARP1 wild-type SUM149 xenografts do. Top panels show Kaplan-Meier survival curves, with a tumour volume of $1000 \mathrm{~mm}^{3}$ (after which mice were sacrificed) used as a surrogate end point. Lower panels show volume of individual tumours, normalised according to tumour volume on the day of randomisation (indicated by the arrow). e Pools of PARP1 guides cause talazoparib resistance in the BRCA1 mutant ovarian cancer cell line COV362. Cas9-expressing COV362 cells were transduced with the indicated lentiviral guide pool, selected in puromycin and assayed as in $\mathbf{c}$. f Silencing of residual BRCA1 function, or BRCA2, induces synthetic lethality with PARP1 genetic loss in SUM149 cells. B1.S* is a SUM149 derivative with a secondary BRCA1 mutation that confers PARP inhibitor resistance. SRB-stained 24well plate image is shown, 7 days after siRNA transfection. $\mathbf{g}$ Colony forming assay using SUM149-Cas9 parental cells and the talazoparib-resistant daughter clone, TR2. Cells were transfected as in f, plated on 6-well plates and colonies stained and counted 2 weeks later. siRNA targeting BRCA1 or $B R C A 2$ significantly reduces survival in PARP1 mutant cells (TR2) compared to the parental SUM149 line ( $t$-test). $\mathbf{h} B R C A 1$ and BRCA2 silencing is also synthetically lethal in COV362 PARP1 mutant clones A4 (p.119_120delKS) and D1 ( $p .848$ delY/YK). Colony formation assay as in $\mathbf{g}, p$ values for $t$-test shown. Mean of three replicates plotted, error bars show SD 
Table 3 PARP1 mutations identified in talazoparib-resistant SUM149 and COV362 clones, determined by Sanger sequencing

\section{Clone SgRNA PARP1 mutation(s)}

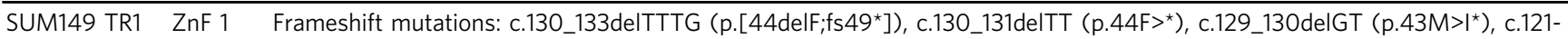

4_130_delCTAGTCGCCCATGTT [splice acceptor]

SUM149 TR2 ZnF 1 p.43delMFD and frameshift mutations: c.130_133delTTTG (p.[44delF;fs49*]), c.129_130insC (p.44F>L*), c.129delG $\left(p .43 M>f s 49^{*}\right)$.

COV362 A4 Pool 2 p.119delKS

COV362 D1 Pool 5 p.848delY/848delYK

COV362 D4 Pool 5 p.848delYKPF

COV362 D8 Pool 5 Wild type

COV362 D9 Pool 5 p.848delY/848delYK

COV362 D11 Pool 5 p.848delY/848delYK

See also Supplementary Data 4 for more detail regarding SUM149 clones

fs frameshift resulting in the indicated number of different amino-acid residues

*Stop codon

BRCA1 exon 11 mutations do not result in complete loss of BRCA1 function ${ }^{24,25}$. Since PARP1;BRCA1 double-mutant cells show distinct drug sensitivities compared to cells that become PARPi resistant via other mechanisms, knowledge of the mechanism of resistance in patients that relapse could inform the best subsequent treatment. Importantly, we also observed a PARP1 mutation that abolished trapping (R591C, Fig. 4f) in a patient with de novo resistance to olaparib, suggesting that such mutations can arise in patients and could potentially contribute to resistance. It also seems reasonable to think that PARPi resistance in some patients might display some level of heterogeneity, with multiple different PARPi subclones emerging with distinct mechanisms of resistance; recent advances in the genomic profiling of both solid and liquid biopsies derived from PARPiresistant patients ${ }^{44-46}$ might potentially assess whether this is the case. Whether this turns out to be the case or not, our observation that PARPi-resistant cells with different mechanisms of resistance display different chemotherapy sensitivities (Supplementary Figure 6) suggests that defining the molecular features of PARPiresistant disease biopsies might be important to determine the best course of subsequent treatment.

The "tag-mutate-enrich" approach we have used, where the tagging of genes with C-terminal GFP coding sequences (or other selectable genes) followed by the targeted mutagenesis of these genes via CRISPR-Cas9 mediated mutagenesis, allows, in principle, full-length mutants of any gene of interest associated with a selectable phenotype to be identified. This could be employed in the analysis of other resistance mutations observed in patients being treated with targeted therapies in order to annotate likely drivers and passengers of resistance.

\section{Methods}

Cell lines. Mouse ES cells were cultured in Knockout DMEM containing 15\% fetal bovine serum (FBS) and leukaemia inhibitory factor as previously described ${ }^{47}$. HeLa, CAL51 (source: DSMZ), COV362 (source: ECACC) and MDA-MB-436 (source: ATCC) cells were maintained in Dulbecco's modified Eagle's medium (DMEM) supplemented with $10 \%$ FBS (with $10 \mu \mathrm{g} / \mathrm{ml}$ insulin in the case of MDAMB-436). The HeLa cell line expressing PARP1-GFP gene from a bacterial artificial chromosome has been previously described ${ }^{48}$. SUM149PT cells (referred to as SUM149, source: Asterand Bioscience) were maintained in Ham's F-12 medium supplemented with $5 \% \mathrm{FBS}, 10 \mu \mathrm{g} / \mathrm{ml}$ insulin and $1 \mu \mathrm{g} / \mathrm{ml}$ hydrocortisone.

All human cell line identities were confirmed by STR typing and verified free of mycoplasma infection using Lonza MycoAlert.

Genome-wide mouse CRISPR screen. We screened a previously described library of mouse ES cells infected with a lentiviral single guide RNA (sgRNA) library targeting 19,150 mouse genes (average five sgRNAs/gene ${ }^{49}$. Two million CRISPR mutagenised cells were exposed to a normally lethal concentration $(25 \mathrm{nM})$ of the clinical PARPi talazoparib ${ }^{17}$ (a.k.a. BMN 673; Fig. 1a) for 6 days and allowed to form colonies. Colonies were picked and expanded in 96-well plates. sgRNA sequences from resistant clones were amplified using U6-F and CRISPR-scaf-R primers. Parp1 genotyping of resistant clones coming from the screen was done with Parp1_CRISPRseq-F and Parp1_CRISPRseq-R primers (Supplementary Data 8) using ReadyMix Taq polymerase (Sigma) and the following conditions: $94^{\circ}$ C for $30 \mathrm{~s} ; 30$ cycles of $94^{\circ} \mathrm{C}$ for $10 \mathrm{~s} ; 56^{\circ} \mathrm{C}$ for $10 \mathrm{~s}$ and $72^{\circ} \mathrm{C}$ for $30 \mathrm{~s}$; and the final extension, $72{ }^{\circ} \mathrm{C}$ for $4 \mathrm{~min}$.

Genome-wide CRISPR screen in SUM149 cells. SUM149-Cas9 cells were generated by transduction of SUM149 cells with a Cas9-bsd lentivirus and selection in $7 \mu \mathrm{g} / \mathrm{ml}$ blasticidin. Cells were infected at multiplicity of infection (MOI) 0.3 with a previously published genome-wide human lentiviral CRISPR library ${ }^{27}$. Cells were selected with puromycin and then placed under talazoparib selection at a concentration that killed all non-infected SUM149 cells ( $100 \mathrm{nM})$. Twelve surviving colonies were picked and analysed for the presence of sgRNA sequences by PCR and Sanger sequencing as above.

Genetically engineered cell lines. CAL51 $P A R P 1^{-/-}$cells were generated using the Edit-R Gene Engineering System (GE Dharmacon). Cells were seeded at a density of $1 \times 10^{5}$ cells/well in 24 -well plates. After $24 \mathrm{~h}$, cells were transfected with $1 \mu \mathrm{g}$ Edit-R CRISPR-Cas9 Nuclease Expression Plasmid mixed with $2.5 \mu \mathrm{l}$ of $20 \mu \mathrm{M}$ PARP1 T2 crRNA (GACCACGACACCCAACCGGAGUUUUA-

GAGCUAUGCUGUUUUG) and $2.5 \mu \mathrm{l}$ of tracrRNA $(20 \mu \mathrm{M})$, using Lipofectamine 3000 according to the manufacturer's instructions (Life Technologies). Four days after transfection, cells were selected in $100 \mathrm{nM}$ talazoparib for 5 days, and surviving cells FACS-sorted into 96-well plates at one cell per well in drug-free medium. After 2 weeks, the medium was changed to $100 \mathrm{nM}$ talazoparib and cells kept under selection for another month. Targeted genome modifications were analysed by Sanger sequencing of PCR products cloned into pCR-TOPO-blunt (Life Technologies). Ten individual colonies were sequenced. Clone T2.4, referred to as CAL51 PARP1 ${ }^{-1-}$, lacks PARP1 expression by western blot and has bi-allelic out-of-frame deletions at the target site: a $50 \mathrm{bp}$ deletion and a single base " $\mathrm{C}$ " deletion, respectively.

Stable Cas9-expressing human cell lines were generated by transducing cells with a Cas9-blasticidin lentivirus ${ }^{50}$ and selecting cells with integrated virus by culturing in medium containing $7 \mu \mathrm{g} / \mathrm{ml}$ blasticidin.

SUM149 TR1 and TR2 were generated by infecting SUM149-Cas9-expressing cells with a pLentiGuide-puro ${ }^{50}$ vector expressing an sgRNA targeting ZnF1 (Hs_PARP1_DBD_cr) and selecting cells with $100 \mathrm{nM}$ talazoparib for 7 days. Single cells were sorted using FACS and the resulting clones genotyped by PCR with Hs_PARP1DBD-genoF and Hs_PARP1DBD-genoR primers (Supplementary Data 8) using Q5 High-Fidelity DNA Polymerase (NEB) with the following conditions: $98^{\circ} \mathrm{C}$ for $30 \mathrm{~s} ; 30$ cycles of $98^{\circ} \mathrm{C}$ for $10 \mathrm{~s} ; 63^{\circ} \mathrm{C}$ for $10 \mathrm{~s}$ and $72^{\circ} \mathrm{C}$ for 30 $\mathrm{s}$; and the final extension, $72^{\circ} \mathrm{C}$ for $2 \mathrm{~min}$.

SUM149 PARP1-tagGFP2 cells were generated by CRISPaint ${ }^{40}$ using as a genespecific target sgRNA sequence 5'-GCAATTTTAAGACCTCCCTG-3'. GFPpositive single-cell clones were isolated. They were genotyped with the Hs_PARP1_intron22_F and tagGFP2_R primers $\left(98^{\circ} \mathrm{C}\right.$ for $30 \mathrm{~s}, 30$ cycles of $98^{\circ} \mathrm{C}$ for $10 \mathrm{~s}, 65^{\circ} \mathrm{C}$ for $10 \mathrm{~s}$ and $72{ }^{\circ} \mathrm{C}$ for $30 \mathrm{~s}$, and the final extension, $72{ }^{\circ} \mathrm{C}$ for $2 \mathrm{~min}$ ), and the PCR product was Sanger sequenced to ensure a single in-frame integration. Clones 5 and 8 originated from independent integration events.

Tdg mutant mouse ES cell clones were generated from JM8A3 (C57BL/6N) cells ${ }^{47}$ by infection with a single lentivirus expressing a $T d g$ targeting sgRNA (Tdg_gRNA) and Cas9 (lentiCRISPR ${ }^{51}$ ). Colonies were picked and genotyped by PCR with Tdg_CR2_checkF and Tdg_CR2_checkR primers (Supplementary Data 8) using ReadyMix Taq polymerase (Sigma) and the following conditions: $94^{\circ}$ C for $30 \mathrm{~s} ; 30$ cycles of $94^{\circ} \mathrm{C}$ for $10 \mathrm{~s} ; 56^{\circ} \mathrm{C}$ for $10 \mathrm{~s}$ and $72{ }^{\circ} \mathrm{C}$ for $30 \mathrm{~s}$; and the final extension, $72^{\circ} \mathrm{C}$ for $4 \mathrm{~min}$. 
a

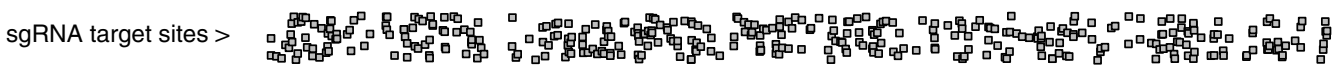

b

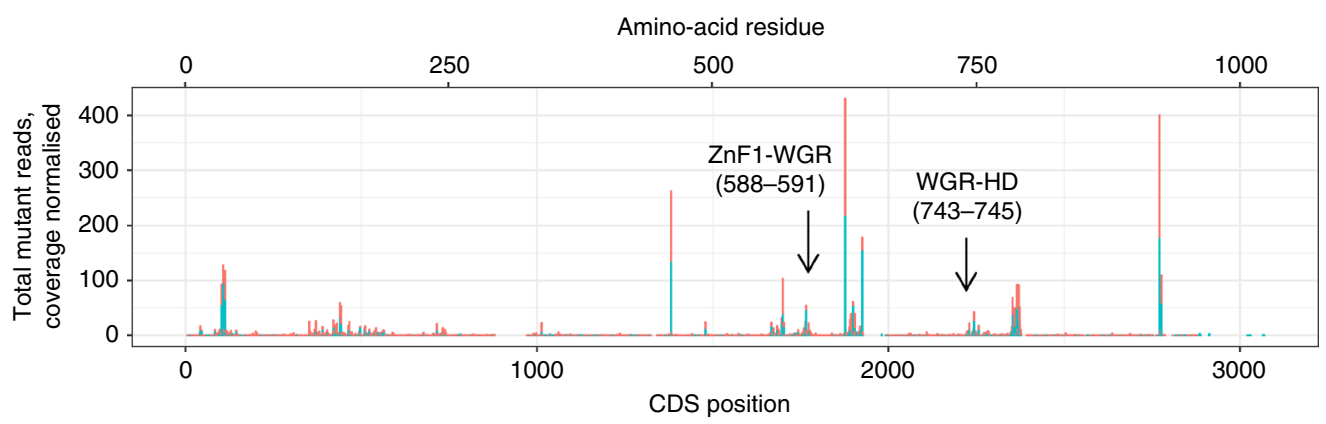

C PARP1 protein domains $-\mathrm{ZnE1}=\mathrm{ZnF2}-\mathrm{ZnF3}$
Intramolecular contacts
DNA-contacting residues
Patient mutation R591C

d

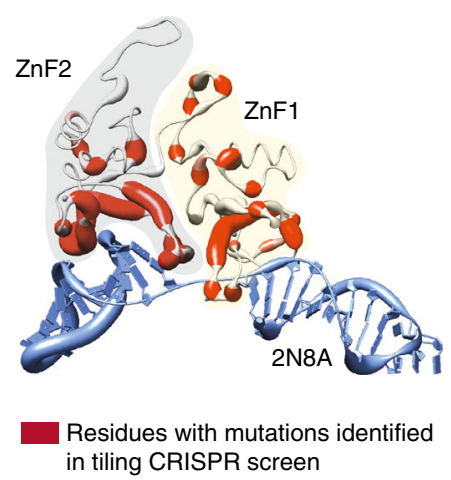

$\mathbf{f}$

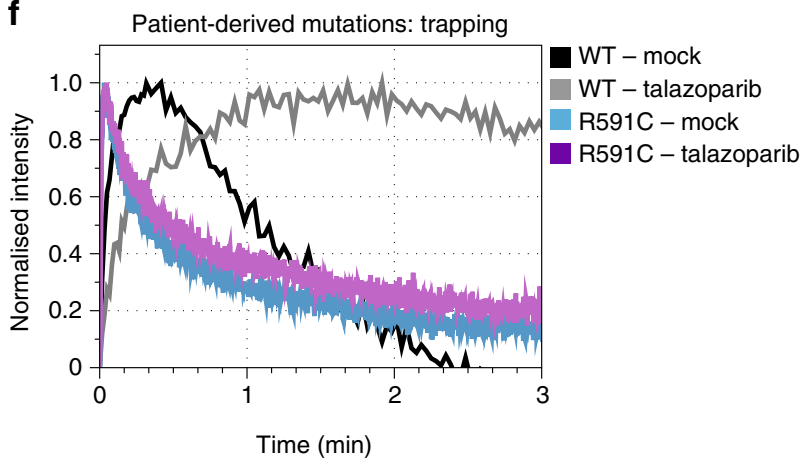

e

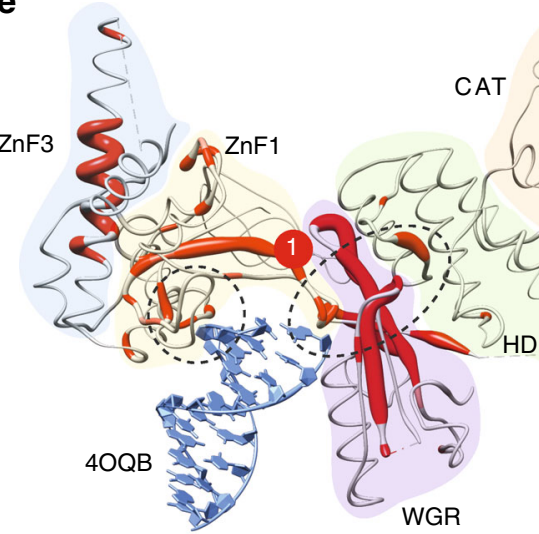

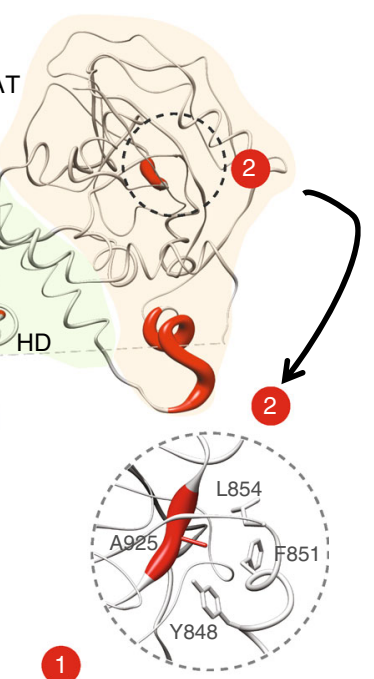

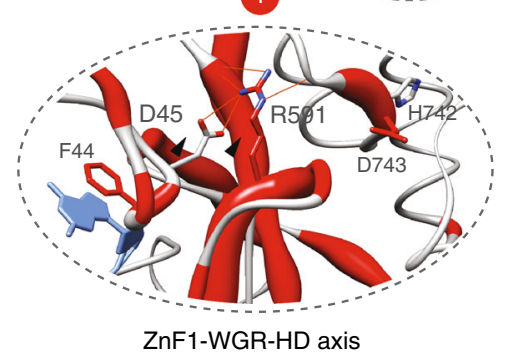

Fig. 4 A high-density tiling CRISPR-Cas9 screen enables functional annotation of a PARP1 mutation observed in an olaparib-resistant patient. a Positions of sgRNA target sites in the dense PARP1 tiling library mapped onto the PARP1 coding DNA sequence shown in $\mathbf{b}$. $\mathbf{b}$ Positions of mutations identified in the dense PARP1 tiling screen. Number of mutant reads for in-frame mutant alleles affecting each base is shown on the $y$-axis normalised to per-base coverage (mutant reads/total coverage $\times 1000$ ). Only sites with coverage $>500$ reads are shown. The experiment was repeated in duplicate from two independently tagged SUM149 PARP1-GFP cell lines (clone 5 and 8, shown in red and blue). Arrows highlight the mutations observed at inter-domain contacts as shown in e. c Positions of protein domains mapped onto the PARP1 protein sequence. Red triangles indicate DNA-protein contacts based on crystal structures, blue circles show the location of the patient mutation identified in this study. Grey arcs represent key inter-domain contacts as shown in e and Fig. $2 \mathrm{~g}$. $\mathbf{d}$ Ribbon plot of ZnF1 and ZnF2 bound to a single-strand break (PDB: 2N8A). Ribbons are coloured red by residue based on the frequency of mutations observed in the tiling screen affecting that residue as shown in $\mathbf{b}$. The thickness of the ribbon is proportional to the frequency of mutations observed affecting that residue. e Ribbon plot of the PARP1-DSB crystal structure (PDB: 4OQB) highlighting the clustering of mutations along the hydrogen-bonding axis postulated in Fig. $2 \mathrm{~g}$ and the $\mathrm{A} 925$ residue that abuts Y848 (inset 2). Regions marked 1 and 2 are magnified. $\mathbf{f}$ Analysis of trapping for the R591C patient mutation by microirradiation with or without talazoparib as shown. Although the R591C mutant can bind DNA at sites of damage (blue line), it is not trapped in the presence of talazoparib (purple line) 
Reagents. Drugs, including olaparib and talazoparib, were purchased from Selleckchem, except temozolomide and methyl methanesulfonate (Sigma-Aldrich). Antibodies used are listed in Supplementary Data 9.

Cell viability and clonogenic assays. The viability of cells was measured after 5 days exposure to various concentrations of drugs using the Cell Titre-Glo assay (Promega). Long-term drug exposure effects were assessed by colony formation assay after 7-10 days exposure to a drug as described previously ${ }^{1}$, with drugcontaining medium refreshed weekly and cells stained at the end of the assay with sulforhodamine B. When plotting survival curves, the surviving fraction was calculated relative to DMSO (solvent)-exposed cells.

PARP1 sgRNA screen in HeLa cells. In all, 29 sgRNAs were designed using the ChopChop algorithm ${ }^{52}$. The guides were synthesised and cloned in an array format into pLentiGuide-puro ${ }^{50}$ by Eurofins genomics. A single colony for each vector was used to inoculate a pooled culture for plasmid maxi prep (Qiagen). Six such pools of vectors were packaged into viruses as previously described ${ }^{53}$. These pooled viruses were used to infect HeLa PARP1-GFP/Cas9 cells. sgRNA-expressing cells were enriched with a 3-day puromycin selection $(3 \mu \mathrm{g} / \mathrm{ml})$. The cells were subsequently incubated in $1 \mu \mathrm{M}$ talazoparib for 12 additional days. The GFP-positive population was enriched by cell sorting (BD FACSAria). Total RNA was extracted from each of the six pools. One microgram of total RNA was converted to cDNA with SuperScript III (ThermoFisher) and a GFP-specific primer (5'-

CCTTGATGCCGTTCTTCTGCTTG-3'), which allowed for amplification only of the target PARP1-GFP transcripts. Each of the six pooled cDNAs was amplified with its corresponding Ion Torrent adapted primer pair flanking the gRNA target sites for that pool. This amplification used Q5 High-Fidelity DNA Polymerase (NEB) with the following PCR conditions: $98^{\circ} \mathrm{C}$ for $30 \mathrm{~s} ; 30$ cycles of $98^{\circ} \mathrm{C}$ for $10 \mathrm{~s}$; $64{ }^{\circ} \mathrm{C}$ for $10 \mathrm{~s}$ and $72^{\circ} \mathrm{C}$ for $30 \mathrm{~s}$; and the final extension, $72^{\circ} \mathrm{C}$ for $2 \mathrm{~min}$. Primer sequences are provided in Supplementary Data 8.

PARP1 dense screen in SUM149 cells. A dense CRISPR library comprising 489 guides targeting PARP1 was synthesised (Twist Biosciences, Supplementary Data 4) and cloned into the BbsI site of pKLV5-U6gRNA5-PGKPuroBFP27. One million cells from each of two SUM149 PARP1-tagGFP2 clones were infected at MOI 0.3 as above and selected in puromycin. Cas9 was induced by treatment with doxycycline for 2 days after which cells were replated and $100 \mathrm{nM}$ talazoparib added. cDNA was prepared using a GFP primer as above and 15 overlapping amplicons prepared by PCR with Ion Torrent adapter tailed primers for genotyping (Supplementary Data 5).

Sequencing and analysis. Purified RT-PCR products were mixed and sequenced using the Ion Torrent PGM and a 318 chip with 850 flows. Data were converted to FASTQ format and aligned to the PARP1 cDNA sequence (ENST00000366794) using Novoalign (Novocraft technologies). Mutations were called from the align ments using the Ensembl Variant Effect Predictor REST API ${ }^{54}$ (implementation: github.com/GeneFunctionTeam/bioruby-sam-mutation). Sequences were also translated and multiple alignments generated using Clustal Omega (http://www. ebi.ac.uk/Tools $/ \mathrm{msa} /$ clustalo/). For the dense screen, coverage was calculated per base using samtools pileup (github.com/samtools, v1.5) with a maximum depth of 300,000 .

Microirradiation assays. Cells were grown in glass-bottom culture dishes (MaTek, P35G-0.170-14-C) in 10\% FBS DMEM media and maintained at $37^{\circ} \mathrm{C}$ and $5 \%$ $\mathrm{CO}_{2}$ in an incubation chamber mounted on the microscope. Imaging was carried out on Andor Revolution system, $\times 60$ water objective with micropoint at $365 \mathrm{~nm}$. Only cells with similar GFP signal intensity were measured. The background intensity (in the vicinity of the microirradiation area in the nucleus) was subtracted from that at the microirradiation point and the maximum was normalised to 1 .

Chromatin fractionation (trapping assay). The chromatin fractionation assay for PARP trapping was based on a previously published protocol ${ }^{6}$. Cells were grown in six-well plates, exposed for $1-4 \mathrm{~h}$ to $500 \mathrm{nM}$ talazoparib and $0.01 \%$ MMS and fractionated with the Subcellular Protein Fractionation kit for Cultured Cells (ThermoFisher \#78840) according to the manufacturer's recommendations. Equivalent volumes of each fraction were analysed by western blot using the PARP1 (Cell Signaling \#9542, rabbit), with TOP1 (BD \#556597, mouse) or Histone H3 (CST \#9717, rabbit; low molecular weight region of blot probed separately) as fractionation controls. Blots were imaged using the LiCor Odyssey multicolour imaging system and secondary antibodies (Donkey anti-rabbit 680 and donkey anti-mouse 800). Uncropped scans of western blots are shown in Supplementary Figure 9.

Protein purification. PARP1 point mutations were introduced in an isopropyl $\beta$-D1-thiogalactopyranoside (IPTG)-inducible C1-PARP1 plasmid (expressing HisMBP-tagged human PARP1 cDNA) and transformed in Rosetta Escherichia coli (Novagen). Cells were grown in terrific broth at $37^{\circ} \mathrm{C}$ until reaching $\mathrm{OD}_{600} 1.0$, $100 \mathrm{mM} \mathrm{ZnSO}_{4}$ was added and cells further grown to $\mathrm{OD}_{600} 2.0$. A unit of $0.5 \mathrm{mM}$
IPTG was added and cells were shaken at $18^{\circ} \mathrm{C}$ overnight. Pellets were collected by centrifugation and stored at $-80^{\circ} \mathrm{C}$. Pellets were lysed $(50 \mathrm{mM}$ Tris- $\mathrm{HCl}(\mathrm{pH} 7.5)$ $500 \mathrm{mM} \mathrm{NaCl}, 10 \mathrm{mM}$ imidazole, $10 \mathrm{mM}$ mercaptoethanol and protease inhibitors), sonicated and cleared by centrifugation and $0.45 \mu \mathrm{m}$ filtration. The lysate was passed through a $5 \mathrm{ml}$ His Trap column (GE). The column was washed and bound proteins eluted with a linear imidazole gradient. Pooled PARP1-containing fractions were dialysed overnight (50 mM Tris- $\mathrm{HCl}(\mathrm{pH} 7.5), 250 \mathrm{mM} \mathrm{NaCl}$ and $5 \mathrm{mM}$ mercaptoethanol) in the presence of MBP-TEV protease. Dialysed proteins were cleared through $0.45 \mu \mathrm{m}$ filter and cleaved MBP and MBP-TEV were removed through another $5 \mathrm{ml}$ His Trap column. Samples were loaded on a $5 \mathrm{ml}$ HiTrap Heparin column (GE) and eluted with a linear salt gradient. PARP1-containing fractions were pooled, concentrated to $1 \mathrm{ml}$ and injected on a HiLoad 16/600 Superdex 200 column (GE) equilibrated in $25 \mathrm{mM}$ HEPES-NaOH (pH 7.5), 100 $\mathrm{mM} \mathrm{NaCl}$ and $2 \mathrm{mM}$ TCEP. PARP1-containing fractions were pooled, concentrated and snap-frozen in liquid $\mathrm{N}_{2}$

PARylation assay. DNA dumbbell ligand (DB4) was prepared as previously described $^{12}$. In vitro PARylation reactions were carried out in a buffer $(50 \mathrm{mM}$ HEPES (pH 7.5), $150 \mathrm{mM} \mathrm{NaCl}, 10 \mathrm{mM} \mathrm{MgCl}_{2}$ and $1 \mathrm{mM}$ dithiothreitol) containing $100 \mathrm{nM}$ PARP1 protein and $1 \mu \mathrm{M}$ DB4. The reaction was started by the addition of $\mathrm{NAD}^{+}$to a $225 \mu \mathrm{M}$ final concentration (spiked with $5 \mu \mathrm{Ci} / \mathrm{ml}^{32} \mathrm{P}_{-\mathrm{NAD}}{ }^{+}$) and incubation at $30^{\circ} \mathrm{C}$. Aliquots were taken at various time points and the reaction was stopped by the addition of $2 \times$ SDS-polyacrylamide gel electrophoresis (SDS-PAGE) loading dye and heat denaturation. Samples were resolved on SDS-PAGE, dried and exposed on a phosphoimager plate (GE). Total radioactive intensity was quantified in the lanes between the well start and the position of PARP1

Xenograft experiment. Xenograft experiments were performed by Crown Bioscience UK. Female BALB/c nude mice aged 6-8 weeks were purchased from Envigo. Mice had access to irradiated G.19\% extruded rodent diet from Harlan Teklad (cat\# 2919) throughout study. To form xenografts $5 \times 10^{6}$ cells were injected subcutaneously into the left flank with 1:1 (v/v) Matrigel as previously described ${ }^{17}$ Tissue from established tumours was pooled and passaged with $25 \mu$ l Cultrex per mouse to new hosts for the treatment study $\left(100 \mathrm{~mm}^{3} /\right.$ mouse, $n=20 /$ cell line). Sample size was determined based on previous experience with this model system. Mice were randomised when average tumour size reached $100 \mathrm{~mm}^{3}$, using the multi-task method in the Studylog software, minimising differences in tumour volume between groups. Talazoparib $(0.66 \mathrm{mg} / \mathrm{ml}$ in DMSO) was diluted in diluent (10\% DMAc and 6\% Solutol in phosphate-buffered saline) and administered orally on a schedule of 3 days on, 4 days off. For the vehicle arm, an equivalent volume of DMSO was diluted as above and administered on the same schedule. Tumour volumes were estimated three times per week using the formula $0.5 \times$ length $\times$ width $^{2}$. For survival analysis, a final tumour limit of $1000 \mathrm{~mm}^{3}$ was used. Blinding was not used. Animal welfare for this study complies with the UK Animals Scientific Procedures Act 1986 in line with Directive 2010/63/EU of the European Parliament and the Council of 22 September 2010 on the protection of animals used for scientific purposes. All experimental data management and reporting procedures were in strict accordance with applicable Crown Bioscience UK Guidelines and Standard Operating Procedures.

siRNA transfection. Lipofectamine RNAimax was used according to the manufacturer's instructions (Invitrogen). Cells were transfected with Smart Pools targeting the appropriate gene (GE Dharmacon).

Patient tumour sequencing. The PARP1:R591C mutation was identified in the archival tumour sample of a 69 -year-old patient (without a BRCA1 or BRCA2 germline mutation) with platinum-resistant high-grade serous ovarian cancer enroled in a phase I trial of olaparib and durvalumab ${ }^{55}$ (NCT02484404). A panel of cancer susceptibility and DNA repair genes (BROCA-HR ${ }^{56}$ ) was sequenced and the PARP1:R591C mutation (genomic: chr1:226 $564979 \mathrm{G}>\mathrm{A}$ ) was identified at a variant allele frequency (VAF) of 0.33 (65/198 reads). This ovarian carcinoma also had a TP53 mutation (p.R196*) with VAF of 0.72 . The patient did not have a response to olaparib and durvalumab based on RECIST v1.1 criteria. The study has been conducted in accordance with ethical principles that have their origin in the Declaration of Helsinki and are consistent with the International Council on Harmonization guidelines on Good Clinical Practice, all applicable laws and regulatory requirements, and all conditions required by a regulatory authority and/or institutional review board. The study protocol was reviewed and approved by the Institutional Review Board of the Center for Cancer Research, National Cancer Institute. Written informed consent was obtained from all patients ${ }^{55}$.

Data availability. Data and cell lines are available from the authors on request. Sequencing data are deposited at the ENA, accession number PRJEB24332.

Received: 29 September 2017 Accepted: 22 March 2018

Published online: 10 May 2018 


\section{References}

1. Farmer, H. et al. Targeting the DNA repair defect in BRCA mutant cells as a therapeutic strategy. Nature 434, 917-921 (2005).

2. Bryant, H. E. et al. Specific killing of BRCA2-deficient tumours with inhibitors of poly(ADP-ribose) polymerase. Nature 434, 913-917 (2005).

3. Lord, C. J. \& Ashworth, A. PARP inhibitors: synthetic lethality in the clinic. Science 355, 1152-1158 (2017).

4. Mortusewicz, O., Amé, J.-C., Schreiber, V. \& Leonhardt, H. Feedbackregulated poly(ADP-ribosyl)ation by PARP-1 is required for rapid response to DNA damage in living cells. Nucleic Acids Res. 35, 7665-7675 (2007).

5. Murai, J. et al. Stereospecific PARP trapping by BMN 673 and comparison with olaparib and rucaparib. Mol. Cancer Ther. 13, 433-443 (2014).

6. Murai, J. et al. Trapping of PARP1 and PARP2 by clinical PARP inhibitors. Cancer Res. 72, 5588-5599 (2012).

7. Pettitt, S. J. et al. A genetic screen using the PiggyBac transposon in haploid cells identifies Parpl as a mediator of olaparib toxicity. PLOS ONE 8, e61520 (2013).

8. Durkacz, B. W., Omidiji, O., Gray, D. A. \& Shall, S. (ADP-ribose)n participates in DNA excision repair. Nature 283, 593-596 (1980).

9. Satoh, M. S. \& Lindahl, T. Role of poly(ADP-ribose) formation in DNA repair. Nature 356, 356-358 (1992).

10. Zahradka, P. \& Ebisuzaki, K. A shuttle mechanism for DNA-protein interactions. The regulation of poly(ADP-ribose) polymerase. Eur. J. Biochem. 127, 579-585 (1982)

11. Dawicki-McKenna, J. M. et al. PARP-1 activation requires local unfolding of an autoinhibitory domain. Mol. Cell 60, 755-768 (2015).

12. Eustermann, S. et al. Structural basis of detection and signaling of DNA singlestrand breaks by human PARP-1. Mol. Cell 60, 742-754 (2015).

13. Langelier, M.-F., Planck, J. L., Roy, S. \& Pascal, J. M. Structural basis for DNA damage-dependent poly(ADP-ribosyl)ation by human PARP-1. Science 336, 728-732 (2012).

14. El Khamisy, S. F., Masutani, M., Suzuki, H. \& Caldecott, K. W. A requirement for PARP-1 for the assembly or stability of XRCC1 nuclear foci at sites of oxidative DNA damage. Nucleic Acids Res. 31, 5526-5533 (2003).

15. Lord, C. J., Tutt, A. N. J. \& Ashworth, A. Synthetic lethality and cancer therapy: lessons learned from the development of PARP inhibitors. Annu. Rev. Med. 66, 455-470 (2015).

16. Lord, C. J. \& Ashworth, A. Mechanisms of resistance to therapies targeting BRCA-mutant cancers. Nat. Med. 19, 1381-1388 (2013).

17. Shen, Y. et al. BMN 673, a novel and highly potent PARP1/2 inhibitor for the treatment of human cancers with DNA repair deficiency. Clin. Cancer Res. 19, 5003-5015 (2013).

18. Ali, A. A. E. et al. The zinc-finger domains of PARP1 cooperate to recognize DNA strand breaks. Nat. Struct. Mol. Biol. 19, 685-692 (2012).

19. Hochegger, H. et al. Parp-1 protects homologous recombination from interference by $\mathrm{Ku}$ and Ligase IV in vertebrate cells. EMBO J. 25, 1305-1314 (2006).

20. Murai, J. et al. Rationale for poly(ADP-ribose) polymerase (PARP) inhibitors in combination therapy with camptothecins or temozolomide based on parp trapping versus catalytic inhibition. J. Pharmacol. Exp. Ther. 349, 408-416 (2014).

21. Cortázar, D., Kunz, C., Saito, Y., Steinacher, R. \& Schär, P. The enigmatic thymine DNA glycosylase. DNA Repair 6, 489-504 (2007).

22. Cortázar, D. et al. Embryonic lethal phenotype reveals a function of TDG in maintaining epigenetic stability. Nature 470, 419-423 (2012).

23. Elstrodt, F. et al. BRCA1 mutation analysis of 41 human breast cancer cell lines reveals three new deleterious mutants. Cancer Res. 66, 41-45 (2006)

24. Hill, S. J., Clark, A. P., Silver, D. P. \& Livingston, D. M. BRCA1 pathway function in basal-like breast cancer cells. Mol. Cell. Biol. 34, 3828-3842 (2014).

25. Wang, Y. et al. The BRCA1-11q alternative splice isoform bypasses germline mutations and promotes therapeutic resistance to parp inhibition and cisplatin. Cancer Res. 76, 2778-2790 (2016).

26. Dréan, A. et al. Modelling therapy resistance in BRCA1/2 mutant cancers. Mol. Cancer Ther. 16, 2022-2034 (2017).

27. Tzelepis, K. et al. A CRISPR dropout screen identifies genetic vulnerabilities and therapeutic targets in acute myeloid leukemia. Cell Rep. 17, 1193-1205 (2016).

28. Ter Brugge, P. et al. Mechanisms of therapy resistance in patient-derived xenograft models of BRCA1-deficient breast cancer. J. Natl Cancer Inst. 108, djw148 (2016).

29. Bouwman, P. et al. 53BP1 loss rescues BRCA1 deficiency and is associated with triple-negative and BRCA-mutated breast cancers. Nat. Struct. Mol. Biol. 17, 688-695 (2010)

30. Norquist, B. et al. Secondary somatic mutations restoring BRCA1/2 predict chemotherapy resistance in hereditary ovarian carcinomas. J. Clin. Oncol. 29, 3008-3015 (2011).
31. Bunting, S. F. et al. 53BP1 inhibits homologous recombination in Brca1deficient cells by blocking resection of DNA breaks. Cell 141, 243-254 (2010)

32. Xu, G. et al. REV7 counteracts DNA double-strand break resection and affects PARP inhibition. Nature 521, 541-544 (2015)

33. Cheung, H. W. et al. Inactivation of human MAD2B in nasopharyngeal carcinoma cells leads to chemosensitization to DNA-damaging agents. Cancer Res. 66, 4357-4367 (2006).

34. Mitra, A. K. et al. In vivo tumor growth of high-grade serous ovarian cancer cell lines. Gynecol. Oncol. 138, 372-377 (2015).

35. Shakya, R. et al. BRCA1 tumor suppression depends on BRCT phosphoprotein binding, but not its E3 ligase activity. Science 334, 525-528 (2011).

36. Berchuck, A. et al. Frequency of germline and somatic BRCA1 mutations in ovarian cancer. Clin. Cancer Res. 4, 2433-2437 (1998).

37. The Cancer Genome Atlas Network. Comprehensive molecular portraits of human breast tumours. Nature 490, 61-70 (2012).

38. Gayther, S. A. et al. Germline mutations of the BRCA1 gene in breast and ovarian cancer families provide evidence for a genotype-phenotype correlation. Nat. Genet. 11, 428-433 (1995).

39. The Cancer Genome Atlas Research Network. Integrated genomic analyses of ovarian carcinoma. Nature 474, 609-615 (2011).

40. Schmid-Burgk, J. L., Höning, K., Ebert, T. S. \& Hornung, V. CRISPaint allows modular base-specific gene tagging using a ligase-4-dependent mechanism. Nat. Commun. 7, 12338 (2016).

41. Herzog, M., et al. Detection of functional protein domains by unbiased genome-wide forward genetic screening. Sci. Rep. 8, 6161 (2018)

42. Xie, S. et al. Timeless interacts with PARP-1 to promote homologous recombination repair. Mol. Cell 60, 163-176 (2015)

43. Young, L. M. et al. TIMELESS forms a complex with PARP1 distinct from its complex with TIPIN and plays a role in the DNA damage response. Cell Rep. 13, 451-459 (2015)

44. Goodall, J. et al. Circulating cell-free DNA to guide prostate cancer treatment with PARP inhibition. Cancer Discov. 7, 1006-1017 (2017).

45. Quigley, D. et al. Analysis of circulating cell-free DNA identifies multiclonal heterogeneity of BRCA2 reversion mutations associated with resistance to PARP inhibitors. Cancer Discov. 7, 999-1005 (2017).

46. Kondrashova, O. et al. Secondary somatic mutations restoring RAD51C and RAD51D associated with acquired resistance to the PARP inhibitor rucaparib in high-grade ovarian carcinoma. Cancer Discov. 7, 984-998 (2017).

47. Pettitt, S. J. et al. Agouti C57BL/6N embryonic stem cells for mouse genetic resources. Nat. Methods 6, 493-495 (2009).

48. Poser, I. et al. BAC TransgeneOmics: a high-throughput method for exploration of protein function in mammals. Nat. Methods 5, 409-415 (2008).

49. Koike-Yusa, H., Li, Y., Tan, E. P., Velasco-Herrera, M. D. C. \& Yusa, K Genome-wide recessive genetic screening in mammalian cells with a lentiviral CRISPR-guide RNA library. Nat. Biotechnol. 32, 267-273 (2013).

50. Sanjana, N. E., Shalem, O. \& Zhang, F. Improved vectors and genome-wide libraries for CRISPR screening. Nat. Methods 11, 783-784 (2014).

51. Shalem, O. et al. Genome-scale CRISPR-Cas9 knockout screening in human cells. Science 343, 84-87 (2014).

52. Montague, T. G., Cruz, J. M., Gagnon, J. A., Church, G. M. \& Valen, E. CHOPCHOP: a CRISPR/Cas9 and TALEN web tool for genome editing. Nucleic Acids Res. 42, W401-407 (2014).

53. Sims, D. et al. High-throughput RNA interference screening using pooled shRNA libraries and next generation sequencing. Genome Biol. 12, R104 (2011).

54. McLaren, W. et al. The Ensembl variant effect predictor. Genome Biol. 17, 122 (2016).

55. Lee, J.-M. et al. Safety and clinical activity of the programmed death-ligand 1 inhibitor durvalumab in combination with poly (ADP-ribose) polymerase inhibitor olaparib or vascular endothelial growth factor receptor 1-3 inhibitor cediranib in women's cancers: a dose-escalation, phase I study. J. Clin. Oncol. 35, 2193-2202 (2017)

56. Walsh, T. et al. Mutations in 12 genes for inherited ovarian, fallopian tube, and peritoneal carcinoma identified by massively parallel sequencing. Proc. Natl Acad. Sci. USA 108, 18032-18037 (2011)

\section{Acknowledgements}

We thank Anita Grigoriadis (BCN Research Unit, King's College, London) for information on PARP1 copy number in SUM149 cells, and Fredrik Wallberg of the ICR, London, for assistance with FACS. This work was funded by Breast Cancer Now and Cancer Research UK Programme Awards to C.J.L. We acknowledge NHS funding to the NIHR Royal Marsden Hospital Biomedical Research Centre. Work in S.G.'s laboratory is supported by The Institute of Cancer Research (ICR), Cancer Research UK through a Career Establishment Award (C47521/A16217) and the Lister Institute of Preventive Medicine. Funding to K.Y. was provided by the Wellcome Trust (WT077187). Funding to S.S. and R.A. was 
provided by the National Science Front of Bulgaria (DFNI-B02/16). Funding was also provided by a Stand Up To Cancer-Ovarian Cancer Research Fund Alliance-National Ovarian Cancer Coalition Dream Team Translational Research Grant (grant number SU2C-AACR-DT16-15 to E.M.S.). Stand Up to Cancer is a programme of the Entertainment Industry Foundation; research grants are administered by the American Association for Cancer Research, a scientific partner of Stand Up To Cancer.

\section{Author contributions}

S.J.P., D.B.K., A.A. and C.J.L. designed the study. S.J.P., D.B.K., I.B., A.D., F.S., R.A., M.I. H. M.M., R.B., J.F., M.R., H.N.P., R.R. and K.F. performed experiments. S.J.P., D.B.K., J. C. and C.J.L. analysed data. M.I.H., J.-M.L. and E.M.S. contributed patient and sequencing data. A.D. and K.Y. contributed reagents. A.S., S.G., S.S., A.A. and C.J.L. supervised the study. S.J.P., D.B.K. and C.J.L. wrote the manuscript.

\section{Additional information}

Supplementary Information accompanies this paper at https://doi.org/10.1038/s41467018-03917-2.

Competing interests: C.J.L. and A.A. are named inventors on patents describing the use of PARP inhibitors and stand to gain from their development as part of the ICR

"Rewards to Inventors" scheme. The remaining authors declare no competing interests.
Reprints and permission information is available online at http://npg.nature.com/ reprintsandpermissions/

Publisher's note: Springer Nature remains neutral with regard to jurisdictional claims in published maps and institutional affiliations.

\section{(c) (i)}

Open Access This article is licensed under a Creative Commons Attribution 4.0 International License, which permits use, sharing, adaptation, distribution and reproduction in any medium or format, as long as you give appropriate credit to the original author(s) and the source, provide a link to the Creative Commons license, and indicate if changes were made. The images or other third party material in this article are included in the article's Creative Commons license, unless indicated otherwise in a credit line to the material. If material is not included in the article's Creative Commons license and your intended use is not permitted by statutory regulation or exceeds the permitted use, you will need to obtain permission directly from the copyright holder. To view a copy of this license, visit http://creativecommons.org/ licenses/by/4.0/.

(C) The Author(s) 2018 